\author{
University of Texas Rio Grande Valley \\ ScholarWorks @ UTRGV
}

Physics and Astronomy Faculty Publications

and Presentations

1998

\title{
Hierarchical search strategy for the detection of gravitational waves from coalescing binaries: Extension to post-Newtonian waveforms
}

Soumya Mohanty

The University of Texas Rio Grande Valley

Follow this and additional works at: https://scholarworks.utrgv.edu/pa_fac

Part of the Astrophysics and Astronomy Commons, and the Physics Commons

\section{Recommended Citation}

Mohanty, S. D. "Hierarchical Search Strategy for the Detection of Gravitational Waves from Coalescing Binaries: Extension to Post-Newtonian Waveforms." Physical Review D, vol. 57, no. 2, American Physical Society, Jan. 1998, pp. 630-58, doi:10.1103/PhysRevD.57.630.

This Article is brought to you for free and open access by the College of Sciences at ScholarWorks @ UTRGV. It has been accepted for inclusion in Physics and Astronomy Faculty Publications and Presentations by an authorized administrator of ScholarWorks @ UTRGV. For more information, please contact justin.white@utrgv.edu, william.flores01@utrgv.edu. 


\title{
Hierarchical search strategy for the detection of gravitational waves from coalescing binaries: Extension to post-Newtonian waveforms
}

\author{
S. D. Mohanty* \\ Inter-University Centre for Astronomy and Astrophysics, Post Bag-4, Ganeshkhind, Pune 411 007, India
}

(Received 21 February 1997; published 22 December 1997)

\begin{abstract}
The detection of gravitational waves from coalescing compact binaries would be a computationally intensive process if a single bank of template wave forms (one step search) is used. In an earlier paper we presented a detection strategy, called a two step search, that utilizes a hierarchy of template banks. It was shown that in the simple case of a family of Newtonian signals, an on-line two step search was $\simeq 8$ times faster than an on-line one step search (for the initial LIGO). In this paper we extend the two step search to the more realistic case of zero spin post ${ }^{1.5}$-Newtonian wave forms. We also present formulas for detection and false alarm probabilities which take statistical correlations into account. We find that for the case of a post ${ }^{1.5}$-Newtonian family of templates and signals, an on-line two step search requires $\sim 1 / 21$ the computing power that would be required for the corresponding on-line one step search. This reduction is achieved when signals having a strength $S$ $=10.34$ are required to be detected with a probability of 0.95 , at an average of one false event per year, and the noise power spectral density used is that of the advanced LIGO. For the initial LIGO, the reduction achieved in computing power is $\sim 1 / 27$ for $S=9.98$ and the same probabilities for detection and false alarm as above. The increase in the efficacy of a two step search in the post ${ }^{1.5}$-Newtonian case comes about chiefly because of an increase in the number of signal parameters since the post ${ }^{1.5}$-Newtonian signal depends on the binary masses $m_{1}$ and $m_{2}$ separately unlike the Newtonian case where only a combination of these masses enters the signal parametrization. The shift to post ${ }^{1.5}$-Newtonian signals also gives rise to some new problems which are not encountered in the analysis of Newtonian wave forms. We describe these problems and take them into account in our analysis. [S0556-2821(98)00404-4]
\end{abstract}

PACS number(s): 04.80.Nn, 95.55.Ym, 95.75.Pq, 97.80.Af

\section{INTRODUCTION}

A radiation reaction driven inspiral of a binary composed of compact massive objects (neutron stars, black holes) would emit gravitational waves that would lie, during the last few minutes before merger, in the sensitive bandwidth of laser interferometric detectors such as the Laser Interferometric Gravitational Wave Observatory (LIGO) [1], VIRGO [2] and GEO600. Even though most such events will produce a signal amplitude well below the noise rms at any given instant, the predictability of such wave forms would allow the use of pattern matching techniques such as matched filtering to considerably improve their chances of detection [3]. In matched filtering, the detector output is passed through a filter that is matched to the expected signal wave form in some optimal sense. If the maximum of the output crosses a pre-determined threshold, a signal is declared to be present in the data with a time of arrival given by the location of the maximum. The filtering of the detector output can, of course, be substituted with a cross correlation against a suitable template wave form that is matched to the expected signal.

Generically, the wave form from an inspiraling binary is an amplitude and phase modulated sinusoid both of whose instantaneous frequency and amplitude increase as the two

\footnotetext{
*Present address: LIGO project, California Institute of Technology, MS 18-34, Pasadena, CA 91125. Email address: mohanty@ligo.caltech.edu
}

bodies proceed towards merger. The signal becomes "visible" in the output of a detector when its instantaneous frequency exceeds the lower frequency cutoff of the output's bandwidth. This moment can be taken as the time of arrival of the signal at the detector. Such a cutoff is required, for instance, in the case of ground based detectors because of excessive seismic noise at low frequencies. There would, of course, be an unknown phase offset at the time of arrival. In addition, the signal would be characterized by the masses and spins of the two components, the distance to the binary and geometrical factors such as the direction to the binary and the orientation of the orbital plane.

Thus, if matched filtering is used for such signals, it would be necessary to employ a bank of filters (or template wave forms) corresponding to different values of the signal parameters mentioned above. One would then compare the maximum over all the filtered outputs with a threshold. This strategy is usually called a one-step search. Even though the time of arrival, initial phase and distance can be handled easily, a one-step search would still be a computationally expensive proposition. Estimates $[4,5]$ of the computational power that is required for an on-line one-step search using post-Newtonian templates turn out to be $\sim 200$ Gflops or higher. This is with the omission of various other signal processing overheads which can be expected to be present in a realistic situation. Therefore, it is desirable to have computationally less expensive detection strategies without, however, compromising too much on the performance afforded by matched filtering. One such strategy, called a two step hierarchical search, was investigated by us in an earlier work [6]. We found that this strategy reduces the computa- 
TABLE I. Minimum $C_{\text {online }}^{(2)}$ as a function of $T$ for $S_{\min }=9.98, \xi_{\max }=140.482 \mathrm{sec}, Q_{\mathrm{d} \text {, } \operatorname{lin}}=0.95, \eta^{(2)}$ $=8.314, l_{1}=0.022 \mathrm{sec}, l_{2}=0.144 \mathrm{sec}, N_{T}^{t}=13279$. The noise power spectral density used is that of the initial LIGO. $C_{\text {online }}^{(2)}$ is the computational power required for an on-line two step search. $T$ is the length used for the detector output segments, $\xi_{\max }$ is the duration of the longest template (corresponding to the binary masses $m_{1}=m_{2}=0.5 M_{\odot}$, the lowest masses used in the analysis), and $S_{\min }$ is the lowest signal strength for which a minimum detection probability of $Q_{\mathrm{d}, \min }$ is required at an average false alarm rate of $1 \mathrm{false}$ event/yr. The lengths of the sides of a unit cell in the second, finer stage of the hierarchy are given by $l_{1}$ and $l_{2}$ which have units of time since the signal parameter space is that of chirp times (see Sec. V for details). $C_{\text {online }}^{(1)}$ is the computational power required if the second stage template grid is used for an on-line one step search $\left(N_{T}^{t}\right.$ being the total number of templates required in that case). The threshold required in the second stage is denoted by $\eta^{(2)}$ and that required in the first stage (at the minimum of $C_{\text {online }}^{(2)}$ ) is denoted by $\eta^{(1)}$. The ratios of the lengths of the sides of a first stage unit cell to the corresponding sides of the second stage unit cell are denoted by $k_{1}$ and $k_{2} . n_{c}^{\mathrm{av}}$ is the average number of false crossings in the first stage (shown zero if $<1$ ) while $n_{t}^{\text {av }}$ is the number of templates used (over both the stages) on the average. $C_{\text {gain }}$ is the ratio $C_{\text {online }}^{(1)} / C_{\text {online }}^{(2)}$.

\begin{tabular}{lcccccccc}
\hline \hline$T(\mathrm{sec})$ & $k_{1}$ & $k_{2}$ & $\eta^{(1)}$ & $n_{c}^{\text {av }}$ & $n_{t}^{\text {av }}$ & $C_{\text {online }}^{(2)}(\mathrm{Gflops})$ & $C_{\text {online }}^{(1)}(\mathrm{Gflops})$ & $C_{\text {gain }}$ \\
\hline 256.0 & 8 & 9 & 6.056 & 0 & 360 & 0.192 & 7.07 & 36.82 \\
512.0 & 8 & 6 & 6.283 & 0 & 441 & 0.155 & 4.65 & 30.00 \\
1024.0 & 8 & 5 & 6.484 & 0 & 490 & 0.152 & 4.12 & 27.11 \\
2048.0 & 8 & 4 & 6.649 & 0 & 620 & 0.187 & 3.99 & 21.34 \\
4096.0 & 8 & 4 & 6.649 & 1 & 682 & 0.207 & 4.02 & 19.42 \\
8192.0 & 8 & 3 & 6.866 & 0 & 733 & 0.228 & 4.12 & 18.07 \\
\hline \hline
\end{tabular}

tional cost of detection significantly without losing out on the performance of a one step search. This is because a two-step search utilizes information that was present in a one-step search but which was neglected, namely, the correlation between templates which allows a coarse scan of the parameter space to predict the location of a threshold crossing peak among the filtered outputs.

Our analysis was restricted in [6] (henceforth referred to as MD96) to the case of Newtonian wave forms and the noise power spectral density used was that of the initial LIGO. The main result of our analysis was that a two step hierarchical search is $\sim 8$ times faster than the corresponding one step search. This gain was achieved when the detection probability desired was 0.95 for a signal to noise ratio of $8.8 \sigma$ at an average rate of false events of $1 / \mathrm{yr}$. The Newtonian template family will, however, not be good enough for the detection of the true signal wave form [7]. It was chosen in MD96 in order to keep the analysis simple since that work was in the nature of a first estimate and several other issues needed to be highlighted. This paper is an extension of the two step search to a more realistic family of signals and templates, namely, the post ${ }^{1.5}$-Newtonian family.

Our choice is motivated by the result of Apostolatos [5] that a post ${ }^{1.5}$-Newtonian template family, having spin parameters $\beta=\sigma=0$, is adequate for the detection of signals up to (and possibly beyond) post $^{2}$-Newtonian order, even for maximally spinning systems. However, this holds for spins that are aligned with the orbital angular momentum. In general, the signal from a misaligned system would suffer significant phase and amplitude modulations that can considerably reduce the signal to noise ratio (SNR), for some detector-binary geometries, if non-spinning templates are used. The larger the opening angle between the orbital and total angular momentum, the larger is the fraction of detector-binary geometries which would be lost. But for moderate opening angles ( $\sim 25^{\circ}$ or less), a sizable fraction of signals can still be detected with the non-spinning tem- plates. Therefore, a non-spinning post ${ }^{1.5}$-Newtonian template family appears to be a realistic one to use. We choose our family of signals also to be the same since, as mentioned above, even higher order signal wave forms may be detectable using this family of templates. This choice of templates and signals should provide a realistic model for the assessment of a two step hierarchical search while keeping the analysis relatively simple. In the following we will refer to non-spinning post ${ }^{1.5}$-Newtonian wave forms as simply post ${ }^{1.5}$-Newtonian ones.

The main result of this paper is summarized in Tables I and II. In Table I, the noise power spectral density (PSD) expected for the initial LIGO [8] has been used, while in Table II, the PSD used is that which is expected for the advanced LIGO [9]. Columns 7 and 8 of each table show the computational power required for an on-line two-step search $\left(C_{\text {online }}^{(2)}\right)$ and an on-line one-step $\left(C_{\text {online }}^{(1)}\right)$ search respectively for the same performance parameters. That is, a detection probability of 0.95 for all signals having a strength $S_{\text {min }}$ (as given in the captions of the tables) and an average false alarm rate of 1 false event/yr. These values of the computational requirement have been obtained for various lengths of the input data segment which are tabulated in the first column. $\xi_{\max }$ is the length of the template having the lowest values for the binary masses, $m_{1}$ and $m_{2}$, which we choose to be $m_{1}=m_{2}=0.5 M_{\odot}$. The highest masses that we have used in our analysis are $\# m_{1}=m_{2}=30.0 M_{\odot}$. These results show that a two step hierarchical search can reduce computational requirements by about a factor of $\sim 25$ in a realistic scenario.

Compared to the Newtonian case, a two step search is faster than a one step search in the case of post ${ }^{1.5}$-Newtonian signals because of an increase in the number of signal parameters. The Newtonian wave form depends on only a combination, called the chirp mass, of the binary masses $m_{1}$ and $m_{2}$. Hence, the template bank is effectively one dimensional. However, the post ${ }^{1.5}$-Newtonian wave form is non-degenrate 
TABLE II. Minimum $C_{\text {online }}^{(2)}$ as a function of $T$ for $S_{\min }=10.34, \xi_{\max }=5621.51 \mathrm{sec}, Q_{\mathrm{d} \text {, min }}=0.95, \eta^{(2)}$ $=8.658, l_{1}=0.116 \mathrm{sec}, l_{2}=0.560 \mathrm{sec}, N_{T}^{t}=300796$. The noise power spectral density used is that of advanced LIGO. For an explanation of the symbols used, refer to the caption of Table I. $\xi_{\max }$ corresponds to the duration of the template with binary masses $m_{1}=m_{2}=0.5 M_{\odot}$ the lowest masses used in our analysis.

\begin{tabular}{lcccccccc}
\hline \hline$T(\mathrm{sec})$ & $k_{1}$ & $k_{2}$ & $\eta^{(1)}$ & $n_{c}^{\text {av }}$ & $n_{t}^{\text {av }}$ & $C_{\text {online }}^{(2)}($ Gflops $)$ & $C_{\text {online }}^{(1)}(\mathrm{Gflops})$ & $C_{\text {gain }}$ \\
\hline 8192.0 & 5 & 9 & 6.649 & 11 & 10188 & 9.771 & 288.48 & 29.52 \\
16384.0 & 4 & 7 & 7.002 & 6 & 13490 & 6.476 & 144.39 & 22.30 \\
32768.0 & 4 & 7 & 7.002 & 16 & 14390 & 5.709 & 119.34 & 20.90 \\
65536.0 & 4 & 7 & 7.002 & 35 & 16100 & 6.014 & 112.36 & 18.68 \\
131072.0 & 4 & 6 & 7.060 & 56 & 18935 & 7.004 & 111.27 & 15.89 \\
\hline \hline
\end{tabular}

in the binary masses and the template bank is two dimensional. The total computational gain in a two step search is then given roughly by the product of the two step gain along each dimension (which is about the same as in the Newtonian case). At the same time, however, an increase in the dimensionality leads to a larger number of templates and hence a larger false alarm for a given threshold. This reduces the computational advantage somewhat and, hence, it is not straightforward to obtain the computational reduction in the post ${ }^{1.5}$-Newtonian case by any simple extrapolation of the Newtonian case. The same reason also prevents a simple extrapolation of results obtained with the initial LIGO power spectral density to the case of the advanced LIGO.

In the Newtonian case, the spacing of templates turns out to be uniform because of the location independence of the intrinsic ambiguity function [6]. In the case of (zero-spin) post-Newtonian wave forms at orders higher than 1.5, the location independence property of this quantity is lost. This makes the estimation of the number of templates more difficult in the latter case. Also, in the Newtonian case, the parameter space was effectively one-dimensional (time of arrival and initial phase being easy to handle). This further simplifies the counting of templates. In the case of zero-spin post ${ }^{1.5}$-Newtonian signals the paramater space is two dimensional and, for the choice of the post-Newtonian chirp times as parameters, the shape of the astrophysically relevant region of parameter space is somewhat non-trivial and complicates the counting of templates. Both these issues are addressed in detail in the present paper and we have attempted to take their effects into account in our final results. It should be emphasized here that when going over to higher order zero-spin post-Newtonian wave forms, essentially the same problems will be encountered without the addition of anything fundamentally different. Thus, the results obtained from an in-depth analysis of the post ${ }^{1.5}$-Newtonian case would also hold to a large extent for higher order wave forms. This is also another reason behind our choice of the post $^{1.5}$-Newtonian wave form in the present paper.

In MD96, the formulas used for detection and false alarm probabilities used the assumption of statistical independence between certain random variables. This assumption fails when templates are placed very closely and we were, thus, limited in exploring the small spacing case more thoroughly. In the present paper, we present a much improved formula for the detection probability that reproduces the Monte Carlo results quite well. It also suggests an alternative approach to Monte Carlo simulations for parameter estimation which should be orders of magnitude faster than the conventional approach but further investigations in this direction are postponed to a later work. We also show, somewhat qualitatively, that the assumption of statistical independence is justified as far as the false alarm is concerned. Thus, the results that we have obtained can be considered to be fairly accurate within the approximations that have been made due to other reasons, like the non-trivial boundary of the space of interest and the location dependence of the intrinsic ambiguity function.

The rest of the paper is organized as follows. In Sec. II we apply the method of maximum likelihood detection to the post ${ }^{1.5}$-Newtonian family of signals. This is the rigorous formalism behind the matched filtering algorithm mentioned above. We end this section with the derivation of a test statistic whose value determines the choice between detection and non-detection. In Sec. III, we study the probability distribution functions of the test statistic. Formulas for the detection and false alarm probability are derived. In Sec. IV the problem of optimaly placing templates in a one-step search is investigated. We obtain some approximations regarding the placement geometry, number of templates etc. Sec. V is devoted to the two-step hierarchical search and also contains the results of this paper. We conclude with Section VI.

\section{MAXIMUM LIKELIHOOD DETECTION OF POST $^{1.5}$-NEWTONIAN SIGNALS}

The method of maximum likelihood detection entails the maximization of the posterior probability $p(x(t) \mid \Theta)$ over the signal parameters $\Theta$. Here, $x(t)$ is a segment of the output of a detector and $p(A \mid B)$ denotes the conditional probability of $A$ given $B$. Actually, one should maximize $p(\Theta \mid x(t))$ because it is $x(t)$ which is given, but when our a priori knowledge of the frequencies with which various values of the parameters can occur is negligible, this is equivalent to the maximization of $p(x(t) \mid \Theta)$. The maximum is then compared with a fixed threshold and a detection is announced if the threshold is crossed. Maximum likelihood detection also achieves the highest average detection probability (averaged over $\Theta$ ) for a given false alarm probability. Actually, this statement is only true approximately but the approximation becomes better as the signal to noise ratio becomes larger [10].

If the detector noise is assumed to be a stationary Gaussian process, defined by a (one-sided) power spectral density $S_{n}(f)$, the above maximization reduces to the maximization, over $\Theta$, of the quantity 


$$
\int_{-\infty}^{\infty} \frac{d f}{S_{n}(f)} \widetilde{x}(f) \tilde{s}^{*}(f ; \Theta)-\frac{1}{2} \int_{-\infty}^{\infty} \frac{d f}{S_{n}(f)} \widetilde{s}(f ; \Theta) \tilde{s}^{*}(f ; \Theta),
$$

where a tilde stands for the Fourier transform of the corresponding time domain function and $s(t ; \Theta)$ stands for a member of the signal family. This motivates the definition of an inner product,

$$
\langle u(t), v(t)\rangle=\int_{-\infty}^{\infty} \frac{d f}{S_{n}(f)} \widetilde{u}(f) \widetilde{v}^{*}(f),
$$

and a corresponding norm

$$
\|u\|=[\langle u, u\rangle]^{1 / 2} .
$$

Thus, maximum likelihood detection involves the computation of

$$
\Lambda=\max _{\Theta}\left[\langle x(t), s(t ; \Theta)\rangle-\frac{1}{2}\langle s(t ; \Theta), s(t ; \Theta)\rangle\right] .
$$

This quantity is known as the test statistic. The test statistic is then compared with a pre-determined threshold to decide whether the given $x(t)$ contains a signal or not. It should be noted that the properties of stationarity and Gaussianity are only approximations for the noise that will be present in the interferometric detectors. However, these approximations are expected to be quite good and we will assume such a noise in the following.

Some clarification should be made here regarding the meaning of a detector output in the context of interferometric gravitational wave detectors. The final output at the photodetector would contain the response of the detector (as calculated using the geodesic deviation equation) convolved with the detector's transfer function [11] (which depends on the way the detector is configured, i.e., the kind of recycling used, the reflectivities of mirrors etc.). This signal would be buried in noise that would be white Gaussian noise if photon shot noise were the only source of noise. So, strictly speaking, the detection strategy should be for the detection of this convolved signal in white noise. However, it is easy to prove that this is equivalent to the detection of the deconvolved signal in a noise which has a power spectral density $S_{n}(f)$ such that when this combination is "passed" through a noise free interferometer, the output is the convolved signal and white noise, as would be observed actually. Clearly, $S_{n}(f)$ should be inversely proportional to the modulus squared of the detector's transfer function. When the noise at the output is not white, as would be the case in practice, $S_{n}(f)$ would be the power spectral density of the actual noise divided by the modulus squared of the detector's transfer function. Henceforth, by the detector output we would mean the deconvolved output which would have noise with a power spectral density given by $S_{n}(f)$ and the signal would be just the bare response of the interferometer, i.e., the relative strain produced in the two arms as computed using the geodesic deviation equation.

\section{A. Post ${ }^{1.5}$-Newtonian signal}

In the case of coalescing binary signals expressed up to the post ${ }^{1.5}$-Newtonian order (spin parameter $\sigma=0$ ), the signal parameters are $\Theta=\left(\mathcal{A}, t_{a}, \Phi, \tau_{0}, \tau_{1.5}\right)$. The parameter $\mathcal{A}$ is the (nearly) constant part of the amplitude of the signal that takes into account the distance to the binary and the relative orientation of the detector and the $T T$ coordinate frames. The rapid rise of power in the seismic noise towards lower frequencies would require that the detector output be bandpass filtered with a cutoff at some low frequency (usually assumed to be $40 \mathrm{~Hz}$ for the initial LIGO and $10 \mathrm{~Hz}$ for the advanced LIGO). Similarly, a cutoff $f_{c}$ will also be required at the high frequency end because of a rise in photon shot noise. Usually, $f_{c}$ is taken as $f_{c}=1000 \mathrm{~Hz}$. Thus, loosely speaking, the signal wave form from an inspiraling binary would "start" in the output of the detector at the time when its instantaneous frequency crosses $f_{a}$. This instant is called the time of arrival of the signal and is denoted by $t_{a}$. The phase of the wave form at $t=t_{a}$ is denoted by $\Phi$. The remaining parameters have the dimension of time and depend on the masses of the binary components. They have been called intrinsic parameters of the wave form in contrast to $t_{a}$ and $\Phi$ which are known as extrinsic parameters.

Actually, the post ${ }^{1.5}$-Newtonian wave form should be characterized by three intrinsic parameters, $\left(\tau_{0}, \tau_{1}, \tau_{1.5}\right)$. The subscripts of $\tau$ denote the post-Newtonian order at which that parameter occurs. Thus, $\tau_{0}$ is the Newtonian chirp time characterizing the lowest order wave form obtained using the quadrupole formalism and the post ${ }^{1}$-Newtonian wave form would have $\tau_{0}$ and $\tau_{1}$ as its intrinsic parameters. However, we have assumed the spins of the binary components to be zero and, hence, we are left with only two independent intrinsic parameters which we have chosen as $\tau_{1.5}$ and $\tau_{0}$ because they can be easily inverted to obtain the masses. In the expression for the wave form, however, we retain $\tau_{1}$ with the understanding that it is dependent on $\tau_{1.5}$ and $\tau_{0}$. In an $a p$ proximate sense, $\tau_{0}+\tau_{1}-\tau_{1.5}$ can be taken to be the time left to the final merger of the binary, starting from $t=t_{a}$.

In the following we will deal with the restricted form of the post ${ }^{1.5}$-Newtonian signal in which post-Newtonian corrections are applied to only the phase of the signal. The restricted wave form at any post-Newtonian level is expected to be a good model for the correct wave form at the same level [9]. The restricted post ${ }^{1.5}$-Newtonian signal is

$$
h(t ; \Theta)=\mathcal{A} a\left(t-t_{a} ; \tau_{0}\right) \cos \left[\int_{-\infty}^{t-t_{a}} d t^{\prime} f\left(t^{\prime} ; \tau_{0}, \tau_{1.5}\right)+\Phi\right],
$$

where

$$
a(t)=\left(1-\frac{t}{\tau_{0}}\right)^{-1 / 4}
$$

and $f\left(t ; \tau_{0}, \tau_{1.5}\right)$, the instantaneous frequency of the signal, is given by an implicit equation

$$
t=\tau_{0}+\tau_{1}-\tau_{1.5}-\left(\tau_{0} x^{-8 / 3}+\tau_{1} x^{-2}-\tau_{1.5} x^{-5 / 3}\right),
$$

where $x=f\left(t ; \tau_{0}, \tau_{1.5}\right) / f_{a}$. The chirp times are given by the following expressions $(G=c=1)$ : 


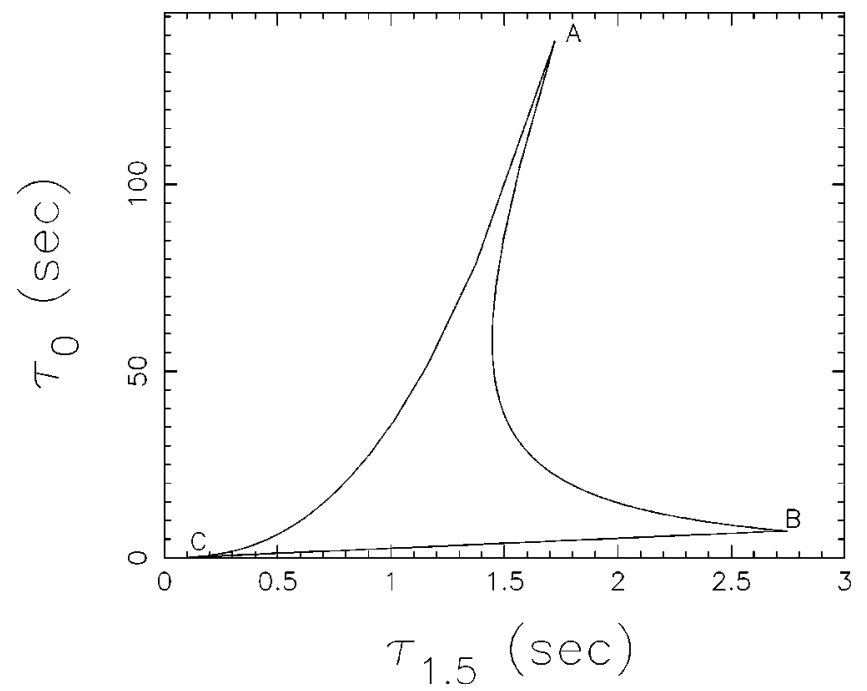

FIG. 1. The space of interest for the case of the initial LIGO noise power spectral density $\left(f_{a}=40.0 \mathrm{~Hz}\right)$. The vertices of the space of interest correspond to the following points in the $\left(m_{1}, m_{2}\right)$ plane: $A$ corresponds to $(0.5,0.5) M_{\odot}, B$ to $(30.0,0.5) M_{\odot}$ and $C$ to $(30.0,30.0) M_{\odot}$.

$$
\begin{gathered}
\tau_{0}=\frac{5}{256} \mathcal{M}^{-5 / 3}\left(\pi f_{a}\right)^{-8 / 3}, \\
\tau_{1}=\frac{5}{192 \mu\left(\pi f_{a}\right)^{2}}\left(\frac{743}{336}+\frac{11}{4} \eta\right), \\
\tau_{1.5}=\frac{1}{8 \mu}\left(\frac{M}{\pi^{2} f_{a}^{5}}\right),
\end{gathered}
$$

where $M$ is the total mass of the binary, $\mu$ is the reduced mass, $\eta=\mu / M$ and $\mathcal{M}=\left(\mu^{3} M^{2}\right)^{1 / 5}$ is the chirp mass. We have chosen $\tau_{0}$ and $\tau_{1.5}$ as our independent parameters. In terms of these parameters,

$$
\begin{gathered}
\tau_{1}=\frac{3715}{4032}\left(\frac{4}{5 \pi^{2}}\right)^{1 / 3} \tau_{0}^{1 / 3} \tau_{1.5}^{2 / 3}+\frac{11}{24 f_{a}} \tau_{0} \tau_{1.5}^{-1}, \\
\mu=\frac{1}{16 f_{a}^{2}}\left(\frac{5}{4 \pi^{4} \tau_{0} \tau_{1.5}^{2}}\right)^{1 / 3}, \\
M=\frac{5}{32} \frac{\tau_{1.5}}{\pi^{2} \tau_{0} f_{a}} .
\end{gathered}
$$

Note that if $\tau_{1}$ were used instead of $\tau_{1.5}$, then the inverse relations for $m_{1}$ and $m_{2}$ would be more complicated and would have to be solved numerically.

In Fig. 1 and Fig. 2, we have shown the $\left(m_{1}, m_{2}\right)$ plane (the binary masses), $0.5 M_{\odot} \leqslant m_{1}, m_{2} \leqslant 30 M_{\odot}$, mapped into the $\left(\tau_{1.5}, \tau_{0}\right)$ space for $f_{a}=40 \mathrm{~Hz}$ (initial LIGO) and $f_{a}$ $=10 \mathrm{~Hz}$ (advanced LIGO) respectively. The boundaries of the region, which we call the space of interest following Apostolatos [5], can be obtained easily. The equation for the curve $A C$, corresponding to $m_{1}=m_{2}$ or $M=4 \mu$, can be found by directly substituting for $\mu$ and $M$ from Eqs. (11) and (12). To obtain the other two segments, $A B$ and $C B$, we use the following expression:

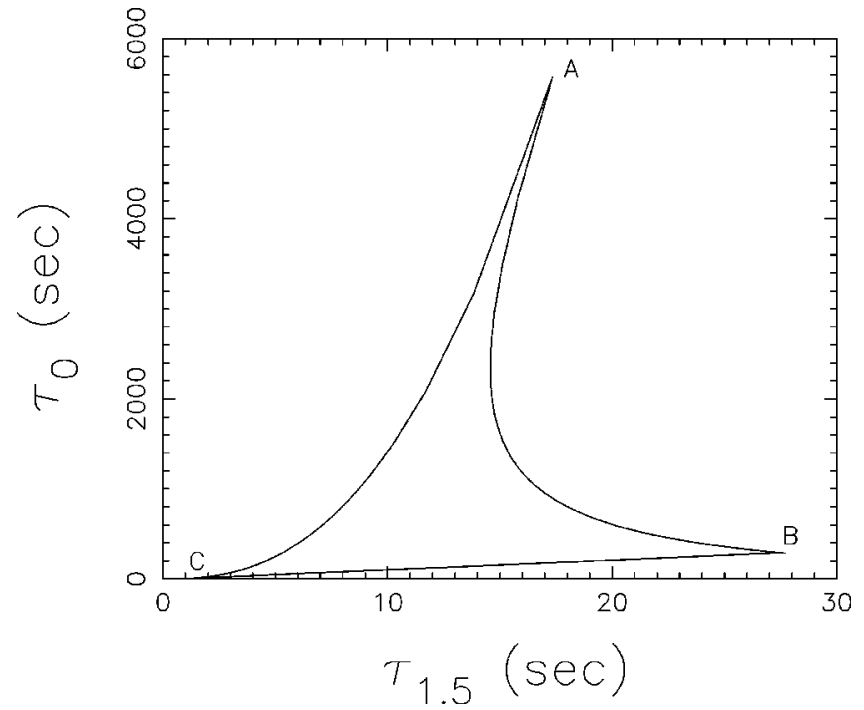

FIG. 2. The space of interest for the case of the advanced LIGO noise power spectral density $\left(f_{a}=10.0 \mathrm{~Hz}\right)$. The vertices of the space of interest correspond to the following points in the $\left(m_{1}, m_{2}\right)$ plane: $A$ corresponds to $(0.5,0.5) M_{\odot}, B$ to $(30.0,0.5) M_{\odot}$ and $C$ to $(30.0,30.0) M_{\odot}$.

$$
\frac{256}{5} \tau_{0}\left(\pi f_{a}\right)^{8 / 3}=\frac{\left(m_{1}+m_{2}\right)^{1 / 3}}{m_{1} m_{2}} .
$$

For every value of $\tau_{0}$, the line of constant $\tau_{0}$ will intersect $A B$ at a point where one of the masses, say $m_{2}$, is $0.5 M_{\odot}$. Similarly, for $B C$, one of the masses, say $m_{1}$, would be $30.0 M_{\odot}$ at the point of intersection. The point where $A B$ and $B C$ meet falls on the $\tau_{0}=\tau_{0}\left(30.0 M_{\odot}, 0.5 M_{\odot}\right)$ line. Thus, for values of $\tau_{0}$ larger than this, one of the masses in the above equation can be set to $0.5 M_{\odot}$ and the equation can be solved for the other mass to yield the value for $\tau_{1.5}$ at the point of intersection. For smaller values of $\tau_{0}$, one of the masses should be set to $30 M_{\odot}$ and $\tau_{1.5}$ be obtained as before. Thus, given a value of $\tau_{0}$, the two limits of $\tau_{1.5}$ can be computed. This allows the area of the space of interest $A$ to be computed using a standard 2D quadrature algorithm (we use D01DAF of the NAg library). The area of the space of interest thus obtained is

$$
A= \begin{cases}50.174 \mathrm{sec}^{2} & \text { for initial LIGO, } \\ 20389.542 \mathrm{sec}^{2} & \text { for advanced LIGO. }\end{cases}
$$

We can write the right-hand side (RHS) of Eq. (4) as

$$
\begin{gathered}
h(t ; \Theta)= \\
\mathcal{A}_{0}\left(t-t_{a} ; \tau_{0}, \tau_{1.5}\right) \cos \Phi \\
+\mathcal{A} h_{\pi / 2}\left(t-t_{a} ; \tau_{0}, \tau_{1.5}\right) \sin \Phi \\
h_{0}\left(t ; \tau_{0}, \tau_{1.5}\right)=a\left(t ; \tau_{0}\right) \cos \left(\int_{-\infty}^{t} d t^{\prime} f\left(t^{\prime} ; \tau_{0}, \tau_{1.5}\right)\right) \\
h_{\pi / 2}\left(t ; \tau_{0}, \tau_{1.5}\right)=a\left(t ; \tau_{0}\right) \cos \left(\int_{-\infty}^{t} d t^{\prime} f\left(t^{\prime} ; \tau_{0}, \tau_{1.5}\right)+\frac{\pi}{2}\right)
\end{gathered}
$$

This representation will be useful in what follows. 
The Fourier transform of $h(t ; \Theta)$ can be computed using the stationary phase approximation. For our purpose, it suffices to give its overall form here, the details being available in other sources $[5,9]$. For $f>0$,

$$
\widetilde{h}(f ; \Theta) \propto \mathcal{A} f^{-7 / 6} \exp \left[-2 \pi i\left(f t_{a}+\sum_{i} \tau_{i} \psi_{i}(f)\right)+i \Phi\right],
$$

where the index $i \in\{0,1,1.5\}$. The functions $\psi_{i}$ are independent of the signal parameters. For $f<0$, the transform is constructed using the Hermitian property of the Fourier transform, $\widetilde{h}(f)=\widetilde{h}^{*}(-f)$, since $h(t, \Theta)$ is a real function.

\section{B. Test statistic and its computation}

Following the brief outline given earlier, the maximum likelihood detection strategy for post ${ }^{1.5}$-Newtonian signals would consist of the computation of a test statistic $\Lambda$ given by

$$
\Lambda=\max _{\Theta}[\langle x(t), h(t ; \Theta)\rangle-1 / 2\langle h(t ; \Theta), h(t ; \Theta)\rangle] .
$$

For the sake of convenience in the following, we adopt the notation

$$
\begin{gathered}
\theta^{\prime}=\left(t_{a}, \tau_{1.5}, \tau_{0}\right), \\
\theta=\left(\tau_{1.5}, \tau_{0}\right)
\end{gathered}
$$

Occasionally, we will also break up $\theta^{\prime}$ as $t_{a}$ and $\theta$. The maximization over the parameters $\mathcal{A}$ and $\Phi$ can be carried out analytically to yield

$$
\Lambda=\max _{\theta^{\prime}} \frac{\left\langle x, q_{0}\right\rangle^{2}+\left\langle x, q_{\pi / 2}\right\rangle^{2}-\left\langle x, q_{0}\right\rangle\left\langle x, q_{\pi / 2}\right\rangle\left\langle q_{0}, q_{\pi / 2}\right\rangle}{1-\left\langle q_{0}, q_{\pi / 2}\right\rangle^{2}},
$$

where

$$
\begin{aligned}
q_{0}(t ; \theta) & =\mathcal{N}_{0}^{-1} h_{0}(t ; \theta), \\
q_{\pi / 2}(t ; \theta) & =\mathcal{N}_{\pi / 2}^{-1} h_{\pi / 2}(t ; \theta), \\
\mathcal{N}_{0} & =\left\|h_{0}(t ; \theta)\right\|, \\
\mathcal{N}_{\pi / 2} & =\left\|h_{\pi / 2}(t ; \theta)\right\| .
\end{aligned}
$$

$\mathcal{N}_{0}$ and $\mathcal{N}_{\pi / 2}$ are normalization constants that are chosen as above for later convenience. Since $t_{a}$ occurs in the phase of the Fourier transforms of $h_{0}\left(t-t_{a} ; \theta\right)$ and $h_{\pi / 2}\left(t-t_{a} ; \theta\right), \mathcal{N}_{0}$ and $\mathcal{N}_{\pi / 2}$ are independent of $t_{a}$. We call $q_{0}(t ; \theta)$ and $q_{\pi / 2}(t ; \theta)$ collectively the template located at $\theta$ and $q_{0}, q_{\pi / 2}$ themselves as the "quadrature" components of the template.

It can be shown, using the stationary phase Fourier transform given in Eq. (18), that

$$
\begin{aligned}
\mathcal{N}_{0} & =\mathcal{N}_{\pi / 2}, \\
\left\langle q_{0}, q_{\pi / 2}\right\rangle & =0 .
\end{aligned}
$$

In general, if

$$
\mathcal{N}_{\Phi}=\|h(t ; \mathcal{A}=1, \Phi, \theta)\|,
$$

then it can be shown using Eq. (18) that

$$
\mathcal{N}_{\Phi}=\mathcal{N}_{0}=\mathcal{N}_{\pi / 2}
$$

Note that $\mathcal{A}=1$ in the above. Equations (28), (30) also hold to a very good approximation when the numerically computed Fourier transforms of the wave forms are used (the typical variation in $\mathcal{N}_{\Phi}$ is $<1 \%$ over $\Phi=[0,2 \pi]$ ). By this we mean that first the wave forms are generated in the time domain using Eq. (6) and then the Fourier transforms are computed using a fast Fourier transform (FFT). Henceforth, we take $\mathcal{N}_{\Phi}$ to be independent of $\Phi$ and denote it simply by $\mathcal{N}$. However, $\mathcal{N}$ does depend on the chirp times via the proportionality constant in Eq. (18).

At this point it is convenient to define a quantity which we call the strength $S$ of a signal $[12,6]$,

$$
S=\mathcal{A N} \text {. }
$$

Henceforth, we use $S$ instead of $\mathcal{A}$ to parametrize a signal. This quantity is essentially the same as the signal to noise ratio $[S / N]$ as defined in [9].

As a consequence of Eq. (27) and Eq. (28), the test statistic in Eq. (19) reduces to

$$
\begin{aligned}
\Lambda & =\max _{\theta^{\prime}}\left[C_{0}^{2}\left(x ; \theta^{\prime}\right)+C_{\pi / 2}^{2}\left(x ; \theta^{\prime}\right)\right]^{1 / 2}, \\
C_{0}\left(x ; \theta^{\prime}\right) & =\left\langle x(t), q_{0}\left(t-t_{a} ; \theta\right)\right\rangle, \\
C_{\pi / 2}\left(x ; \theta^{\prime}\right) & =\left\langle x(t), q_{\pi / 2}\left(t-t_{a} ; \theta\right)\right\rangle .
\end{aligned}
$$

The square root in Eq. (32) is, strictly speaking, not necessary but we retain it in order to make our analysis conform to some of the existing literature.

It has not been possible so far to analytically proceed further with the maximization involved in Eq. (32) and recourse must be taken to numerical techniques. Several numerical methods are available for the maximization of functions [13]. The simpler ones among such methods, though very fast, tend to fail quite badly when confronted with a function involving many local maxima (as would be the case here). For such functions, these methods have a tendency to converge on one of the local maxima rather than the required global maximum. There exist more sophisticated numerical methods such as simulated annealing [13], genetic algorithms and some recent methods such as Price's controlled random search [14]. However, it is very difficult to quantify the performance of such methods in terms of false alarm and detection probabilities. This is a necessity for gravitational wave searches of binary inspirals, and also for other sources in general, given the very small expected event rate. For these reasons, most of the attention in the literature has been directed towards a grid based search for the maximum which is simple enough that its performance can be analyzed theoretically to a large extent. The practical implementation of such a method, called a one-step search, can be motivated as follows.

For a fixed $\theta=\theta_{0}$, the computation of $C_{0}\left(x ; t_{a}, \theta_{0}\right)$ [or $\left.C_{\pi / 2}\left(x ; t_{a}, \theta_{0}\right)\right]$, as a function of $t_{a}$, is equivalent to taking the linear correlation [15] of $x(t)$ with the template $q_{0}\left(t ; \theta_{0}\right)$ 
(or $q_{\pi / 2}$ in the case of $C_{\pi / 2}$ ). Since correlations can be computed efficiently using the FFT [16], the maximization over $t_{a}$ alone is straightforward: The detector output would be sampled at a rate greater than or equal to the Nyquist rate (in this case $\sim 2000 \mathrm{~Hz}$ ), yielding a discrete time series $\bar{x}$ $=\left(x_{0}, x_{1}, \ldots, x_{N-1}\right)$. This time series can then be correlated, using FFTs, with analogous time series $\bar{q}_{0}$ and $\bar{q}_{\pi / 2}$ for the two quadrature components. However, the whole output of such a correlation cannot be used since the use of a FFT will yield a circular correlation instead of a linear one. It is, therefore, necessary to have a padding of zeros at the end of each template time series. Let the duration of this padded part be $T_{P}$ sec for some template. Then, for that template, only the first $T_{P}$ sec of the correlation outputs will be the result of a linear correlation and the rest would have to be discarded. Note that $T_{P}$ will depend on the template parameters since the duration of the wave form is parameter dependent (approximately equal to $\tau_{0}+\tau_{1}-\tau_{1.5}$ ). Let the longest duration among the template wave forms be $\xi_{\max }$ sec (not to be confused with the Newtonian chirp time used in MD96). Then, the shortest linear correlation will have a duration of $T_{P}^{0}=T-\xi_{\max }$, where $T$ is the duration of the of the input time series $\bar{x}$. We will assume in this paper that only the first $T_{P}^{0}$ sec will be kept in each correlation output even if the duration of a template wave form is much less than $\xi_{\max }$. This appears as a wasteful procedure and a better use of computational resources may be possible. However, we do not investigate this issue here since it is not directly relevant to this paper.

Having obtained the correlations with $\bar{q}_{0}$ and $\bar{q}_{\pi / 2}$, the first $T_{P}^{0}$ sec of each correlation should be squared, corresponding samples of the two outputs should be added and the square root taken to yield a single time series. We call this final time series obtained for some template parameters $\theta$ the rectified output [6] of the template $\theta$. Such rectified outputs can be constructed for several points in the $\left(\tau_{1.5}, \tau_{0}\right)$ space and the maximum found over each of them. Finally, the maximum of all these maxima will yield an approximation to the test statistic $\Lambda$. This, essentially, is the scheme of a one step search. We call the set of points in $\left(\tau_{1.5}, \tau_{0}\right)$ space for which rectified outputs are generated the bank of templates. The coordinates of any rectified output sample are given by $\theta^{\prime}$ while those of a template are given by $\theta$.

\section{DISTRIBUTIONS OF THE TEST STATISTIC}

To quantify the performance of the detection strategy described above, we need to calculate the probability of a false alarm as well as that of detection for a given signal. The former is defined as the probability of the test statistic crossing the threshold when a signal is absent in $x(t)$. The latter is the probability of the test statistic crossing the threshold when a signal is present. It should be noted that the detection probability need not be the same for all signals in a signal family. For instance, large amplitude signals should, obviously, have a larger detection probability than weaker ones. We denote the detection probability of a signal with parameters $\Theta$ by $Q_{d}(\eta ; \Theta)$ where $\eta$ denotes the threshold. We denote the false alarm probability by $Q_{0}(\eta)$. If the probability density function of $\Lambda$, the test statistic, is $p_{0}(\Lambda)$ in the absence of a signal and $p_{1}(\Lambda ; \Theta)$ in the presence of a signal, then

$$
\begin{gathered}
Q_{0}(\eta)=\int_{\eta}^{\infty} p_{0}(\Lambda) d \Lambda, \\
Q_{d}(\eta ; \Theta)=\int_{\eta}^{\infty} p_{1}(\Lambda ; \Theta) d \Lambda .
\end{gathered}
$$

In order to construct $p_{0}$ and $p_{1}$, we start at the lowest level, namely, the density functions of a single sample of a rectified output.

Let $z\left(\theta^{\prime}\right)$ be a sample of some rectified output. Now, this sample will be of the form $z\left(\theta^{\prime}\right)=\left[C_{0}^{2}\left(\theta^{\prime}\right)+C_{\pi / 2}^{2}\left(\theta^{\prime}\right)\right]^{1 / 2}$, where the dependence on $x(t)$ has not been shown explicitly. As mentioned before, both $C_{0}\left(\theta^{\prime}\right)$ and $C_{\pi / 2}\left(\theta^{\prime}\right)$ are obtained from correlations involving the detector output. Thus, they are linear combinations of the samples of $x(t)$ and it follows that their marginal probability density function is a Gaussian since the noise is assumed to be a Gaussian random process. In the presence of a signal $h\left(t ; S, \theta_{s}^{\prime}, \Phi_{s}\right)$, their mean values would be

$$
\begin{gathered}
\bar{C}_{0}\left(\theta^{\prime}\right)=\left\langle h\left(t ; S, \theta_{s}^{\prime}, \Phi_{s},\right), q_{0}\left(t-t_{a} ; \theta\right)\right\rangle, \\
\bar{C}_{\pi / 2}\left(\theta^{\prime}\right)=\left\langle h\left(t ; S, \theta_{s}^{\prime}, \Phi_{s}\right), q_{\pi / 2}\left(t-t_{a} ; \theta\right)\right\rangle,
\end{gathered}
$$

while in the absence of a signal, they will have zero means. With our choice of $\mathcal{N}_{0}$ and $\mathcal{N}_{\pi / 2}$ in Eqs. (25), (26), it can be shown that the variance of $C_{0}\left(\theta^{\prime}\right)$ and $C_{\pi / 2}\left(\theta^{\prime}\right)$ is unity. It can also be shown, using Eq. (28), that $C_{0}\left(\theta^{\prime}\right)$ and $C_{\pi / 2}\left(\theta^{\prime}\right)$ are statistically independent of each other. Note that, in general, the covariance of $C_{0}\left(\theta_{1}^{\prime}\right)$ and $C_{\pi / 2}\left(\theta_{2}^{\prime}\right)$ need not vanish for $\theta_{1}^{\prime} \neq \theta_{2}^{\prime}$. Given these properties for $C_{0}$ and $C_{\pi / 2}$, it follows that the marginal probability density function of a rectified output sample is a $\operatorname{Rician} \operatorname{Ri}(z)$ when a signal is present and a Rayleigh $R(z)$ in the absence of a signal,

$$
\begin{aligned}
& \operatorname{Ri}(z)=z \exp \left[-\frac{1}{2}\left(z^{2}+d^{2}\right)\right] I_{0}(z d), \\
& R(z)=z \exp \left[\frac{z^{2}}{2}\right]
\end{aligned}
$$

where

$$
d^{2}=d^{2}\left(\theta^{\prime}\right)=\bar{C}_{0}^{2}\left(\theta^{\prime}\right)+\bar{C}_{\pi / 2}^{2}\left(\theta^{\prime}\right),
$$

and $I_{0}(x)$ is the modified Bessel function of the first kind (of order zero). For $z d \gg 1$, the asymptotic form of $\operatorname{Ri}(z)$ is,

$$
\operatorname{Ri}(z) \sim \sqrt{\frac{z}{2 \pi d}} \exp \left[-\frac{1}{2}(z-d)^{2}\right] .
$$

Thus, for $z \simeq d$, a Rician density goes over into a Gaussian.

Under the stationary phase approximation of Eq. (18), it can be shown that $d\left(\theta^{\prime}\right)$ is independent of the phase $\Phi$ of the signal. Again, this is a good approximation for the exact numerical case. We assume henceforth that $d\left(\theta^{\prime}\right)$ is independent of the signal phase $\Phi$. Let 


$$
\begin{aligned}
H\left(\theta_{p}^{\prime}, \theta_{q}^{\prime}\right)= & {\left[\left\langle q_{0}\left(t-t_{a}^{p} ; \theta_{p}\right), q_{0}\left(t-t_{a}^{q} ; \theta_{q}\right)\right\rangle^{2}\right.} \\
& \left.+\left\langle q_{0}\left(t-t_{a}^{p} ; \theta_{p}\right), q_{\pi / 2}\left(t-t_{a}^{q} ; \theta_{q}\right)\right\rangle^{2}\right]^{1 / 2} .
\end{aligned}
$$

Since $d\left(\theta^{\prime}\right)$ is almost independent of the signal $\Phi$, it follows from Eqs. (37), (38), and (41) that

$$
d\left(\theta^{\prime}\right)=\operatorname{SH}\left(\theta^{\prime}, \theta_{s}^{\prime}\right) .
$$

The quantity $H\left(\theta_{p}^{\prime}, \theta_{q}^{\prime}\right)$ is related to the ambiguity function [10]. Note that since $t_{a}$ occurs in the phase of a Fourier transform, $H\left(\theta_{p}^{\prime}, \theta_{q}^{\prime}\right)$ depends on $t_{a}^{p}$ and $t_{a}^{q}$ only through $\Delta t_{a}=t_{a}^{p}-t_{a}^{q}$.

In order to obtain the distribution of the test statistic $\Lambda$ we need to know the joint distribution of, in general, all the samples. In the presence of a sufficiently strong signal, however, it can be expected that $\Lambda$ will occur almost always only among those samples of the rectified outputs which have a high value of $d$. For a typical number of samples in a single rectified output of $\sim 10^{5}$, for instance, the location of $\Lambda$ is restricted significantly if some samples have $d \geqslant 5.0$. Otherwise, $\Lambda$ occurs almost randomly anywhere within the output time series. Thus, to obtain the distribution of $\Lambda$ in the presence of a signal, we need to consider only a restricted subset of all the samples, namely, those for which $d \gg 1$. The distribution of $\Lambda$ would then be that of the maximum over this subset. We describe a general scheme for choosing this subset below, but for the present, we assume that it is given. As shown above [see Eq. (42)], each sample of this set would have a marginal distribution that is approximately a Gaussian. It is plausible that the joint distribution of such samples can also be approximated by a multivariate Gaussian. This possibility can be investigated by computing the moments of the joint distribution and comparing them with those of a multivariate Gaussian.

We can express a Rician variable $Z=\sqrt{X_{1}^{2}+X_{2}^{2}}$, where $X_{i}$ is a Gaussian random variable with mean $\mu_{i}$, as

$$
\begin{aligned}
Z & =\left[\left(\mu_{1}+\delta X_{1}\right)^{2}+\left(\mu_{2}+\delta X_{2}\right)^{2}\right]^{1 / 2}, \\
& =\sqrt{\mu_{1}^{2}+\mu_{2}^{2}}\left[1+\frac{2\left(\mu_{1} \delta X_{1}+\mu_{2} \delta X_{2}\right)}{\mu_{1}^{2}+\mu_{2}^{2}}+\frac{\delta X_{1}^{2}+\delta X_{2}^{2}}{\mu_{1}^{2}+\mu_{2}^{2}}\right]^{1 / 2},
\end{aligned}
$$

where $\delta X_{1}$ and $\delta X_{2}$ are zero mean Gaussian random variables with unit variances. In the above expression and in the following, we will follow the customary practice of denoting a random variable by an uppercase letter while denoting its value in a particular realization by the corresponding lowercase letter. The probability that $2\left(\mu_{1} \delta X_{1}+\mu_{2} \delta X_{2}\right)+\delta X_{1}^{2}$ $+\delta X_{2}^{2}$ is larger than $\mu_{1}^{2}+\mu_{2}^{2}$ can be obtained easily,

$$
\begin{aligned}
\operatorname{Pr}\left[\frac{2\left(\mu_{1} \delta X_{1}+\mu_{2} \delta X_{2}\right)+\delta X_{1}^{2}+\delta X_{2}^{2}}{\mu_{1}^{2}+\mu_{2}^{2}} \geqslant 1\right] \\
\quad=\frac{1}{2 \pi} \int_{0}^{2 \pi} d \theta \exp \left[-\frac{1}{2} d^{2}\left(\sqrt{1+\cos ^{2}[\theta]}-\cos [\theta]\right)^{2}\right],
\end{aligned}
$$

where $d^{2}=\mu_{1}^{2}+\mu_{2}^{2}$. In our analysis, $d \sim 7.0$ or larger. For $d$ $=7.0$, the above probability is 0.008 and it decreases rapidly for higher values. Thus, only a small fraction realizations would be such that their binomial expansion in terms of $\left[2\left(\mu_{1} \delta X_{1}+\mu_{2} \delta X_{2}\right)+\delta X_{1}^{2}+\delta X_{2}^{2}\right] /\left(\mu_{1}^{2}+\mu_{2}^{2}\right)$ would be nonconvergent. It would be a good approximation to neglect such realizations and calculate the moments of $Z$ by expanding the RHS of Eq. (45) in a binomial expansion and taking the ensemble average for each term.

The same argument goes through for multivariate moments also except for the fact that the fraction of realizations for which all the components of the moment can be expanded binomially will decrease as the number of different variates increases. For instance, if the third moment $\overline{\left(Z_{1}-a_{1}\right)\left(Z_{2}-a 2\right)\left(Z_{3}-a_{3}\right)}$ is required around some point $\left(a_{1}, a_{2}, a_{3}\right)$, then the fraction of cases for which the above expansion will be invalid, taking $d \sim 7$ for all of them, would be $\sim 0.008 \times 3$. In practice there would be a significant overlap of various cases since the $Z_{i}$ would be statistically correlated and this fraction would actually be less. In any case, for low order moments, this method would still furnish a good approximation since the fraction of realizations with non-convergent expansions is still small. For higher values of $d$, the fraction of invalid expansions would go down rapidly, bringing higher moments also under the purview of this method.

Now consider the restricted subset of rectified output samples mentioned above. Let this set be $\left\{Z_{1}, \ldots, Z_{m}\right\}$ and the mean values of the Gaussian components $\left\{\left(X_{11}, X_{12}\right), \ldots,\left(X_{m 1}, X_{m 2}\right)\right\}$ associated with these samples be $\left\{\left(\mu_{11}, \mu_{12}\right), \ldots,\left(\mu_{m 1}, \mu_{m 2}\right)\right\}$. That is, $Z_{i}=\left[X_{i 1}^{2}+X_{i 2}^{2}\right]^{1 / 2}$ and $\bar{X}_{i 1}=\mu_{i 1}$ and $\bar{X}_{i 2}=\mu_{i 2}$. Let the strength of the signal be $S$. Following the argument given above, we can express a moment (about mean values) of the joint distribution of $\left\{Z_{i}, \ldots, Z_{m}\right\}$ as

$$
\begin{aligned}
\mathrm{E}\left[\left(Z_{1}-d_{1}\right)^{p} \ldots\left(Z_{m}-d_{m}\right)^{s}\right] & \\
= & \mathrm{E}\left\{\left[\frac{\mu_{11} \delta x_{11}+\mu_{12} \delta x_{12}}{d_{1}}\right]^{p} \ldots\left[\frac{\mu_{m 1} \delta x_{m 1}+\mu_{m 2} \delta x_{m 2}}{d_{m}}\right]^{s}\right\} \\
& +\mathrm{E}\left\{O\left[\delta x^{2(p+\ldots+s)} / S\right]\right\},
\end{aligned}
$$

where $\mathrm{E}[]$ denotes an ensemble average. Note that the first term is independent of $S$. Also, since $\delta X_{i 1}$ and $\delta X_{i 2}$ are Gaussian random variables, this term is a moment of a multivariate Gaussian distribution. The remaining terms are inversely dependent on $S$ as is shown schematically above. In general, the lowest order correction to the Gaussian moment will have an $S^{-2}$ dependence for even moments and an $S^{-1}$ dependence for odd moments.

Thus, for a sufficiently high $S$, the moments of the joint distribution function of $\left\{Z_{i}, \ldots, Z_{m}\right\}$ are approximately the same as the moments of a multivariate Gaussian distribution. This implies that for $S \gg 1$, the joint distribution of the set $\left\{Z_{1}, \ldots, Z_{m}\right\}$ is given by an $m$-variate Gaussian distribution. It is not easy to see how small the corrections to the Gaussian parts of the moments should be for a given error in the detection probability. However, the above argument provides a sufficiently strong motivation to proceed with the multivariate Gaussian approximation to the joint distribution of 
$\left\{Z_{1}, \ldots, Z_{m}\right\}$. In order to construct this distribution, we need only compute the covariance matrix for $\left\{Z_{1}, \ldots, Z_{m}\right\}$.

Suppose we have two samples $Z_{i}$ and $Z_{j}$ having coordinates $\theta_{i}^{\prime}=\left(t_{a}^{i}, \tau_{1.5}^{i}, \tau_{0}^{i}\right)$ and $\theta_{j}^{\prime}=\left(t_{a}^{j}, \tau_{1.5}^{j}, \tau_{0}^{j}\right)$ respectively. It is easy to show, using the stationary phase Fourier transform, that the covariance matrix of $\left\{X_{i 1}, X_{i 2}, X_{j 1}, X_{j 2}\right\}$ is, with the columns of the matrix in the same order,

$$
\mathbf{C}=\left(\begin{array}{llll}
1 & 0 & r & s \\
0 & 1 & -s & r \\
r & -s & 1 & 0 \\
s & r & 0 & 1
\end{array}\right),
$$

where

$$
\begin{aligned}
& r=\left\langle q_{0}\left(t-t_{a}^{i} ; \theta_{i}\right), q_{0}\left(t-t_{a}^{j} ; \theta_{j}\right)\right\rangle, \\
& s=\left\langle q_{0}\left(t-t_{a}^{i} ; \theta_{i}\right), q_{\pi / 2}\left(t-t_{a}^{j} ; \theta_{j}\right)\right\rangle .
\end{aligned}
$$

Both $|r|$ and $|s|$ are less than unity. The above form of $\mathbf{C}$ is also approximately true when the numerically computed Fourier transforms of the templates are used. The covariance of $Z_{i}$ and $Z_{j}$ can now be computed using Eq. (47) with the RHS truncated to the first term. We get

$$
\sigma_{i j}=\frac{1}{d_{i} d_{j}}\left[r\left(\mu_{i 1} \mu_{j 1}+\mu_{i 2} \mu_{j 2}\right)+s\left(\mu_{i 1} \mu_{j 2}-\mu_{i 2} \mu_{j 1}\right)\right] .
$$

The same kind of calculation also yields $\sigma_{i}=\sigma_{j}=1$. The covariance matrix, for the set $\left\{Z_{1}, \ldots, Z_{m}\right\}$ above, can now be computed using Eq. (51). We can also express the covariance as $\sigma_{i j}=\sqrt{r^{2}+s^{2}} \chi$, where

$$
\chi=\tan ^{-1}\left[\frac{\mu_{1}}{\mu_{2}}\right]+\tan ^{-1}\left[\frac{\nu_{2}}{\nu_{1}}\right]-\tan ^{-1}\left[\frac{s}{r}\right] .
$$

Note that $\sqrt{r^{2}+s^{2}}$ is precisely the quantity $H\left(\theta_{i}^{\prime}, \theta_{j}^{\prime}\right)$.

We list here the general expressions for the first three multivariate moments obtained using Eq. (47), up to the lowest order correction to the Gaussian part. The algebra involved is tedious but a lot of it was automated using the symbolic computation package MATHEMATICA. Let the three rectified output samples be $Z_{m}=\left[X_{1}^{2}+X_{2}^{2}\right]^{1 / 2}, Z_{n}=\left[Y_{1}^{2}\right.$ $\left.+Y_{2}^{2}\right]^{1 / 2}$ and $Z_{o}=\left[W_{1}^{2}+W_{2}^{2}\right]^{1 / 2}$ and the covariance matrix for $\left(X_{1}, X_{2}, Y_{1}, Y_{2}, W_{1}, W_{2}\right)$ be (columns ordered in the same way)

$$
\mathbf{C}=\left(\begin{array}{llllll}
1 & 0 & r_{1} & s_{1} & r_{2} & s_{2} \\
0 & 1 & -s_{1} & r_{1} & -s_{2} & r_{2} \\
r_{1} & -s_{1} & 1 & 0 & t & u \\
s_{1} & r_{1} & 0 & 1 & -u & t \\
r_{2} & -s_{2} & t & -u & 1 & 0 \\
s_{2} & r_{2} & u & t & 0 & 1
\end{array}\right) .
$$

The moments are

$$
\begin{aligned}
& \overline{\left(Z_{m}-d_{m}\right)}=\frac{1}{2 d_{m}}, \\
& \overline{\left(Z_{m}-d_{m}\right)^{2}}=1-\frac{1}{4 d_{m}^{2}}, \\
& \overline{\left(Z_{m}-d_{m}\right)\left(Z_{n}-d_{n}\right)}=\sigma_{m n}+\left[\frac{1}{4 d_{m} d_{n}}\right. \\
& -\frac{\sigma_{m n}}{2 d_{m}^{2} d_{n}^{2}}\left(d_{m}^{2}+d_{n}^{2}\right. \\
& \left.\left.-\sigma_{m n} d_{m} d_{n}\right)\right] \\
& \overline{\left(Z_{m}-d_{m}\right)^{3}}=\frac{3}{2 d_{m}}, \\
& \overline{\left(Z_{m}-d_{m}\right)^{2}\left(Z_{n}-d_{n}\right)}=\frac{1+r_{1}^{2}+s_{1}^{2}}{d_{n}}+\frac{\sigma_{m n}}{d_{m}}-\frac{1}{2 d_{n}} \\
& -\frac{\sigma_{m n}^{2}}{d_{n}} \\
& \overline{\left(Z_{m}-d_{m}\right)\left(Z_{n}-d_{n}\right)\left(Z_{o}-d_{o}\right)}=\frac{1}{2}\left[-\left(\frac{\sigma_{n o}}{d_{m}}+\frac{\sigma_{m n}}{d_{o}}+\frac{\sigma_{m o}}{d_{n}}\right)\right. \\
& +2\left(\frac{\widetilde{\sigma}_{o m} \widetilde{\sigma}_{n m}}{d_{m}}-\frac{\widetilde{\sigma}_{m n} \widetilde{\sigma}_{o n}}{d_{n}}\right. \\
& \left.\left.+\frac{\widetilde{\sigma}_{o m} \tilde{\sigma}_{o n}}{d_{o}}\right)\right]
\end{aligned}
$$

where

$$
\widetilde{\sigma}_{i j}=\frac{1}{d_{i} d_{j}}\left[r\left(\mu_{i 1} \mu_{j 1}-\mu_{i 2} \mu_{j 2}\right)+s\left(\mu_{i 1} \mu_{j 2}+\mu_{i 2} \mu_{j 1}\right)\right]
$$

Other moments up to third order can be constructed from the above expressions by substituting appropriate indices.

Given a bank of templates and the parameters of a signal, the subset $\left\{Z_{1}, \ldots, Z_{m}\right\}$ can be chosen as follows. Let the coordinate of the signal be $\theta_{s}^{\prime}=\left(t_{a}^{s}, \tau_{1.5}^{s}, \tau_{0}^{s}\right)$. A set of templates is chosen from the template bank which lie in a neighborhood of $\left(\tau_{1.5}^{s}, \tau_{0}^{s}\right)$, where the size of this neighborhood is adjustable. In the rectified output of each of these templates, the sample with the largest value of $d$ is identified. We denote the coordinate of such a sample by $\theta_{\alpha, j}^{\prime}$, where the first index stands for the intrinsic parameters $\left(\tau_{1.5}\right.$ and $\left.\tau_{0}\right)$ of the template and the second stands for the location of the sample within the rectified output of this template. From Eq. (44), it follows that the location of this sample for a given $\alpha$ can be found by maximizing $H\left(\theta_{\alpha, k}^{\prime}, \theta_{s}^{\prime}\right)$ over the time of arrival $k$. For each $\theta_{\alpha, j}^{\prime}$, we also choose $2 n$ neighboring samples in the same rectified output, namely, the samples $\left\{\theta_{\alpha, j+k}^{\prime}\right\}$, where $-n \leqslant k \leqslant n$ and $k \neq 0$. Finally, the set of all these samples, 
namely, the set $\left\{\theta_{\alpha, j}^{\prime}\right\}$ and for each $(\alpha, j)$, the set $\left\{\theta_{\alpha, j+k}^{\prime}\right.$; $-n \leqslant k \leqslant n, k \neq 0\}$, gives us the required subset of rectified output samples. Note that $H\left(\theta_{1}^{\prime}, \theta_{2}^{\prime}\right)$ plays a central role in the determination of this subset. In our analysis we find that for most of the cases, $n=1$ or keeping only the two nearest neighbors to $\theta_{\alpha, j}$ is a good approximation. The choice of the neighborhood of templates is intimately related to the placement of the templates themselves and is the subject of the sections which follow.

The distribution of the test statistic $\Lambda$, in the presence of a signal, is that of the maximum of the set $\left\{Z_{1}, \ldots, Z_{m}\right\}$. The joint distribution of this set was shown above to be well approximated by a multivariate Gaussian when the strength of the signal is sufficiently high. An analytical form for the distribution of the maximum of a set of Gaussian random variables is known only for the bivariate case. There are some approximate methods [17] for the calculation of the distribution but these are impractical for more then four or five variates. However, the distribution for a larger number of variates can be estimated from Monte Carlo simulations. A large number of realizations are generated, and for each realization, the maximum value is recorded and finally an estimate of of the required distribution is obtained.

Given the covariance matrix $\mathbf{C}_{X}$ of a multivariate Gaussian random variable $\bar{X}=\left(X_{1}, \ldots, X_{N}\right)$, a realization of $\bar{X}$ can be generated as follows. Let $\left\{\lambda_{i} ; 1 \leqslant i \leqslant N\right\}$ be the set of eigenvalues of $\mathbf{C}_{X}$. Let $\mathcal{E}_{\text {vec }}$ be an $N \times N$ matrix whose $i$ th column is the eigenvector of $\mathbf{C}_{X}$ corresponding to $\lambda_{i}$. If $\bar{W}$ $=\left(W_{1}, \ldots, W_{N}\right)$ is a zero mean multivariate Gaussian with a covariance matrix given by $\operatorname{diag}(1,1, \ldots, 1)$, then

$$
\left[\begin{array}{c}
x_{1} \\
x_{2} \\
\vdots \\
x_{N}
\end{array}\right]=\mathcal{E}_{\mathrm{vec}}\left[\begin{array}{c}
\sqrt{\lambda_{1}} w_{1} \\
\sqrt{\lambda_{2}} w_{2} \\
\vdots \\
\sqrt{\lambda_{N}} w_{N}
\end{array}\right] .
$$

If $\bar{X}$ has a non-zero mean vector, then this can be added to the RHS of the above expression. It is easy to understand the above expression geometrically. In an $N$ dimensional Cartesian space, realizations of $\bar{W}$ will be distributed in a spherically symmetric “cloud." Multiplying the components of $\bar{W}$ by $\sqrt{\lambda}_{i}$ turns this spehrical distribution into an ellipsoidal one. This is the distribution expected for realizations of $\bar{X}$ in the principal axis frame of $\mathbf{C}_{X}$. Finally, a rotation from the principal axis frame to the actual frame is applied. Another method that can be used [18] is to perform the Cholesky decomposition of $\mathbf{C}_{X}$ with the elements of one of the factors chosen in such a way as to give the correct covariances for the components of $\bar{X}$. We use the method of Eq. (61) in our analysis.

An estimate of the distribution of $\Lambda$ can also be obtained using the kind of Monte Carlo simulation that is conventionally used for studies of parameter estimation accuracy. In such a method, a number of realizations of a noisy detector output time series are generated. For each such data segment, rectified outputs are generated for a set of templates and the location of the maximum over the outputs is recorded. The distributions of the coordinates of the maximum then give an estimate of parameter estimation accuracy. One can also record the values of the maximum and, thus, obtain the distribution of $\Lambda$. Note that a distribution obtained in this way would be free of any approximations.

However, there are some limitations to this method. The first is computational. In a typical simulation in our context, each realization of noisy data would have $\sim 10^{4}$ samples (for a $1.4,1.4 M_{\odot}$ binary), for the case of the initial LIGO, and it would be processed through $\sim 5$ templates. This leads to $\sim 10^{6}$ floating point operations (flop) for each realization [19]. A simulation with $\sim 2000$ realizations would thus involve performing $\sim 2 \times 10^{9}$ flop (we have neglected the cost of generating the noise realization itself). This is not a large requirement computationally, but when the same calculations are repeated for the the advanced LIGO case, for signals having comparable masses, this requirement becomes $\sim 10^{12}$ flop. This is because the duration of a signal with the above masses is $\tau_{0} \sim 10^{3} \mathrm{sec}$ for the case of advanced LIGO. Even on a 300 Mflops machine (where an Mflop is $10^{6}$ flop and "flops" stands for flop/sec), a typical high end computing power, it would take $\sim 1 \mathrm{~h}$ to complete the simulation. In our analysis, detection probabilities would be required for various configurations $\left(\sim 10^{2}\right)$ of template placement and would, thus, be quite impractical to compute using this method. There is also a more fundamental limitation. Pseudo-random number generators have, in general, a finite period [20]. For instance, the basic generator provided in the NAg library of numerical routines has a recommended maximum output of $4.0 \times 10^{8}$ random numbers. For the advanced LIGO case, therefore, it is actually not possible to generate more than $\sim 200$ realizations. This, of course, would lead to very poor statistics.

On the other hand, the method represented by Eq. (61) is extremely fast, and since it does not depend on the signal duration, it is equally applicable to both the initial and advanced LIGO cases. Let the number of samples in the set $\left\{\theta_{\alpha, j}\right\}$ be $M$. Then the total number of samples in the set $\left\{Z_{1}, \ldots, Z_{m}\right\}$ would be $m=M \times(2 n+1)$. Given the covariance matrix for $\left\{Z_{1}, \ldots, Z_{m}\right\}$, the number of operations required to obtain a single realization would be essentially $\mathrm{m}^{2}$. Typically, $M \sim 5$ and $n=2$ which leads to 225 flop per realization, a trivial quantity computationally. Also, since for each realization only 15 samples need to be generated, the number of trials can be made as large as $10^{7}$. However, we find approximately 10000 trials to be sufficient for a convergence of the estimated distribution. Though the computational requirements for the simulation itself are small, there is a hidden cost in this method, namely, the computation of the $\mu_{i}, r$ and $s$. If one were to employ FFTs for their computation, the method would again become time consuming. However, these quantities can be computed quite accurately by using the stationary phase Fourier transform also. This way, the computation of the covariance matrix also becomes quite fast. Typically, the whole simulation including the generation of the covariance matrix takes a few seconds on a 30 Mflops machine. This should be compared with the corresponding numbers obtained above for the conventional method. It should be noted that this method may be used for simulations of parameter estimation accuracy also. Further investigations in this direction are in progress. 
Template and Signal positions

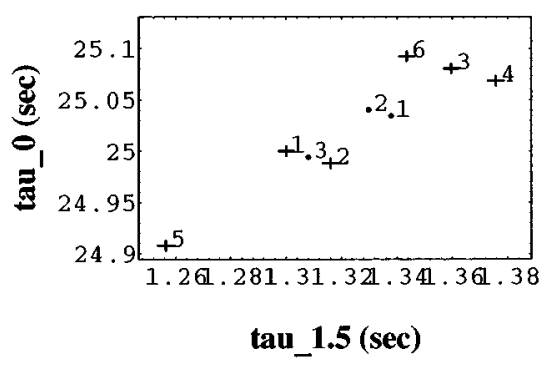

Signal \# 2

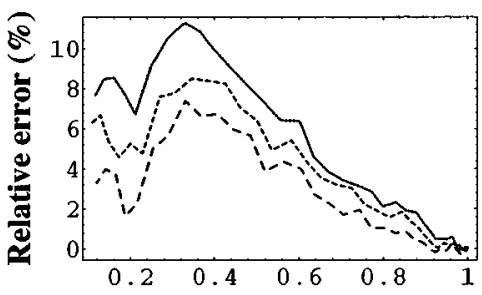

Detection probability
Signal \# 1

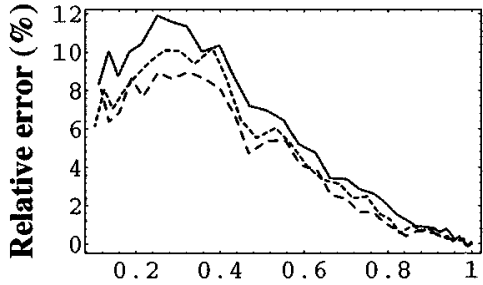

Detection probability

Signal \# 3

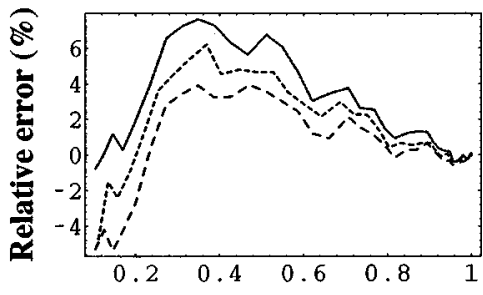

Detection probability

FIG. 3. The relative error in detection probability as obtained using the multivariate Gaussian approximation and as obtained by performing an exact simulation. Each figure shows the relative error for three values of signal strength, $S$ $=8.0$ (solid line), 9.0 (dotted line), 10.0 (dashed line). As expected, the error decreases for larger signal strengths. The top left figure shows the locations of the templates (crosses), used in the calculation of the detection probabilities, and the signal locations (solid circles). The basic unit cell which is composed of templates Nos. 1, 2, 3 and 4 is oriented along the eigenvectors of the Hessian at the location of template No. 1. Templates Nos. 5 and 6 are included in the calculation to take into account any possible contribution that they may provide, because of the shear of contours, to the detection probabilities of signal Nos. 3 and 2, respectively.

In Fig. 3, we compare the performance of the method of multivariate Gaussian with the exact one. It can be seen that the approximate method becomes better as the strength of the signal is increased. In our analysis, a value of 0.95 for the detection probability will be taken as fiducial and, as can be seen from Fig. 3, the error in the approximate method is negligible.

We now turn to the calculation of false alarm probability. An exact expression for the distribution of $\Lambda$ in this case is easily obtained when all the rectified output samples being considered form a statistically independent set. In the presence of statistical correlations between the samples, it appears that an analytical treatment is difficult. However, it was found in MD96 that Monte Carlo estimates of false alarm probability, as a function of threshold, could be fit almost exactly by a formula obtained by assuming that all rectified output samples were statistically independent but there were an effectively lesser number of them. That is, if $Q_{0}(\eta)$ is the probability that the maximum over $N_{r}$ rectified output samples crosses $\eta$ (in the absence of a signal), then

$$
\begin{aligned}
Q_{0}(\eta) & \simeq 1-\exp \left[-\epsilon N_{r} e^{-\eta^{2} / 2}\right] \\
& \approx N_{r} \epsilon \exp \left[-\frac{\eta^{2}}{2}\right] \quad(\text { for } \eta \gg 1),
\end{aligned}
$$

where $0<\epsilon<1$. This fit was assumed to hold for higher values of $\eta$ also though they were beyond the range of the simulations since, as mentioned earlier, the period of pseudorandom number generators is finite and, consequently, it is not possible to generate enough realizations for the estimation of low probabilities. However, we subsequently found that this problem was investigated by Rice [21] (in 1944), though only for the case of a single time series. The formulas obtained in that work can also be interpreted in terms of an effective number of samples but the parameter $\epsilon$ depends on $\eta$ and is not a constant. Specifically, $\epsilon$ approaches unity as the threshold is made higher. Therefore, the extrapolation of the fit to Monte Carlo estimates that was made in MD96 is not valid. This does not affect the results of MD96 significantly, however, because the quantity required in that analysis was $\eta$ for a given false alarm and it was shown to be highly insensitive to $\epsilon$. But it should be noted that this implies that $Q_{0}(\eta)$ is affected significantly by small errors in $\eta$ and, hence, the threshold should be recomputed once the placement of templates is completed.

The extension of the derivation of $Q_{0}(\eta)$ given by Rice (actually, it was the rate of false alarms that was derived) to a random field does not appear to be straightforward. Note that the set of rectified outputs from a given template bank can be considered to be the samples of an underlying 3dimensional random field, one of the dimensions being the time of arrival and the other two being $\tau_{1.5}$ and $\tau_{0}$. We present here a qualitative argument which can be extended to 
the case of random fields also and show that the same conclusion regarding the behavior of $\epsilon$ should hold.

The basis of this argument is the assumption that, in the absence of a signal, any rectified output sample is equally likely to be the location of $\Lambda$. This can be expected to be true provided the random field is at least wide sense stationary. Since the input noise is assumed to be a stationary random process, it follows that the rectified output of any one template will also be stationary. However, the random field can be non-stationary because of non-stationarity in $\tau_{1.5}$ and $\tau_{0}$ or it is genuinely stationary but sampled non-uniformly in $\tau_{1.5}, \tau_{0}$. For the random field to be wide-sense stationary [22], it is required that the correlation of any two samples should depend on only their relative displacement and not their locations.

In the present case, the correlation of any two samples, $Z_{1}$ and $Z_{2}$, can be obtained exactly in the absence of a signal. The derivation given in the Appendix leads to the following expression for the correlation:

$$
\overline{Z_{1} Z_{2}}=2 \mathbf{E}\left[H\left(\theta_{1}^{\prime}, \theta_{2}^{\prime}\right)\right]-\left[1-H\left(\theta_{1}^{\prime}, \theta_{2}^{\prime}\right)^{2}\right] \mathbf{K}\left[H\left(\theta_{1}^{\prime}, \theta_{2}^{\prime}\right)\right],
$$

where $\mathbf{E}$ is the complete elliptic integral of the second kind and $\mathbf{K}$ is the complete elliptic integral of the first kind. Thus, the correlation depends only on $H\left(\theta_{1}^{\prime}, \theta_{2}^{\prime}\right)$. Therefore, if $H\left(\theta_{1}^{\prime}, \theta_{2}^{\prime}\right)$ were location independent, that is, if it were dependent only on the difference $\Delta \theta^{\prime}$ between $\theta_{1}^{\prime}$ and $\theta_{2}^{\prime}$, then the rectified output field would be at least wide sense stationary.

As discussed below, $H\left(\theta_{1}^{\prime}, \theta_{2}^{\prime}\right)$ is location independent in the case of Newtonian and the post ${ }^{1}$-Newtonian wave forms ( $\theta^{\prime}$ is understood to be a different set of parameters for these wave forms) but not in the case of post ${ }^{1.5}$-Newtonian wave form. However, for simplicity, consider the case where $H\left(\theta_{1}^{\prime}, \theta_{2}^{\prime}\right)$ is location independent. We apply our argument to a single rectified output first.

Since we will be using an extremely low false alarm probability in our analysis, the threshold required will be quite high [typically, $(8-9) \sigma$ ]. Suppose that $\eta$ is so high that the probability of two or more simultaneous crossings of $\eta$, at widely separated locations in the time series, is almost zero. By a wide separation we mean roughly that the locations are not closer than the typical correlation length scale. Of course, it is still possible for samples which are highly correlated with the one at which $\Lambda$ occurs to simultaneously cross $\eta$. Thus, if $Z_{i}$ is the sample at which $\Lambda$ occurs (and crosses $\eta$ ), then we have assumed above that the probability of $Z_{j}$ crossing $\eta$ in the same rectified output is zero, where $(|j-i|) \Delta t$ $>L$ and $L$ is the full width at half maximum (FWHM) (say) of the autocorrelation function. We will further assume that $\eta$ is sufficiently high so that if one of the neighboring samples of $Z_{i}$ crosses $\eta$ along with $Z_{i}$, it is almost always either $Z_{i+1}$ or $Z_{i-1}$.

To compute the false alarm probability, we need to count the number of times $\Lambda$ crosses $\eta$ in some $N$ trials for $N \rightarrow \infty$. Under the above assumptions, the number of favorable cases can be counted approximately as follows. First, the number of times a given sample $Z_{i}$ exceeds $\eta$ is counted. For $N$ sufficiently large, it is just the marginal frequency $n$ for that sample

$$
\begin{aligned}
n & =N \times \int_{\eta}^{\infty} d z z \exp \left[-\frac{z^{2}}{2}\right] \\
& =N \times \exp \left[-\frac{\eta^{2}}{2}\right] .
\end{aligned}
$$

Out of these $n$ cases, some would be such in which either $Z_{i-1}$ or $Z_{i+1}$ also cross $\eta$ simultaneously. The number $\tilde{n}$ of such cases in which $Z_{i-1}$ crosses $\eta$ would be $\tilde{n}=n$ $\times p\left(z_{i-1}>\eta \mid z_{i}>\eta\right)$, where $p(A \mid B)$ is the probability of event $A$ given that event $B$ has occurred. The number of cases in which $Z_{i+1}$ crosses $\eta$ simultaneously with $Z_{i}$ would also be $\tilde{n}$. Now, each sample has $n$ events associated with it that favor $\Lambda>\eta$, but out of these, $2 \tilde{n}$ events are common with its immediate neighbors and these common events should be counted only once (recall that, by assumption, the number of events with more than two simultaneous crossings is negligible). Thus, the total number of events $n_{f}$ that favor a false alarm is

$$
\begin{aligned}
n_{f} & \simeq N_{r} \times \tilde{n}+N_{r} \times(n-2 \tilde{n}) \\
& =N_{r}\left[1-p\left(z_{i-1}>\eta \mid z_{i}>\eta\right)\right] \times N \exp \left[-\frac{\eta^{2}}{2}\right],
\end{aligned}
$$

where boundary effects have been neglected. Hence, the false alarm probability is

$$
Q_{0}(\eta)=N_{r}\left[1-p\left(z_{i-1}>\eta \mid z_{i}>\eta\right)\right] \exp \left[-\frac{\eta^{2}}{2}\right]
$$

A comparison of the above with Eq. (63) explains why the latter expression produces a good fit to Monte Carlo estimates but now it can be seen that $\epsilon=p\left(z_{i-1}>\eta \mid z_{i}>\eta\right)$ is not a constant as assumed in MD96 but depends on $\eta$,

$$
\boldsymbol{\epsilon}=\frac{1}{\exp \left[-\frac{\eta^{2}}{2}\right]} \int_{\eta}^{\infty} \int_{\eta}^{\infty} d u d v P_{Z_{i}, Z_{i-1}}(u, v)
$$

where $P_{Z_{i}, Z_{i-1}}(u, v)$ is given in Eq. (A10). Even for such a simple argument, the above expression for $\epsilon$ yields values that are of the same order as those obtained by fitting the Monte Carlo estimates. For instance, from Eq. (68) we get $\epsilon=0.33$ for $\eta=6.0$ and $r^{2}+s^{2}=0.9$. The typical value for $\epsilon$ that was obtained in MD96 was $\sim 0.7$, for a single rectified output.

It is also clear that the assumptions made above regarding simultaneous crossings of $\eta$ are not strictly necessary. The essential point is that simultaneous crossings reduce the number of favorable events and, since the number of such events per sample is identical for all samples (under the assumption of stationarity), this can be expressed as an effective reduction in the number of samples themselves. In this way, the extension of the above argument to a random field is obvious and leads to the same conclusion, namely, that $\epsilon \rightarrow 1$ for $\eta \gg 1$. In this paper, therefore, we use Eq. (62) for the false alarm probability but with $\epsilon=1$. 
In the following, we will need to estimate the threshold that is required to obtain a given false alarm probability. For small values of the false alarm probability, we get, from Eq. (63),

$$
\eta \approx\left(\ln \left[\frac{N_{r}^{2}}{Q_{0}^{2}}\right]\right)^{1 / 2}
$$

Let the number of templates in the template bank be $N_{T}$. Then, $N_{r}=N \times N_{T}$, where $N$ is the number of samples in a single rectified output. Therefore,

$$
\frac{\Delta \eta}{\eta} \approx \frac{\Delta N_{T}}{\eta^{2} N_{T}} .
$$

This shows that the threshold is very insensitive to the number of templates provided the false alarm is kept low. For instance, even if there is a relative error of 50\% in estimating $N_{T}$, the relative error in $\eta$ would just be $\sim 0.8 \%$. As far as the detection probability of a signal is concerned, such an error is entirely negligible. This point will be of importance later in the paper.

\section{PLACEMENT OF TEMPLATES FOR A ONE-STEP SEARCH}

In the previous section, we described a method for the calculation of the detection probability of a given signal. This method consists of choosing a small set of samples $\mathcal{Z}$ from the rectified outputs of templates which are in some neighborhood of the signal. The set $\mathcal{Z}$ is supposed to be such that the maximum, over all rectified output samples, almost always occurs among the members of $\mathcal{Z}$ and this requires that each of them should have a high value of $d$ (typically, $d \geqslant 7.0$ ). Thus, the neighborhood of templates should be such that the maximum of each rectified output, in the absence of noise, be sufficiently large.

This motivates the introduction of a quantity called the intrinsic ambiguity function $\mathcal{H}\left(\tau_{1.5}^{a}, \tau_{0}^{a} ; \tau_{1.5}^{b}, \tau_{0}^{b}\right)$ which is defined as

$$
\mathcal{H}\left(\theta_{a}, \theta_{b}\right)=\max _{\substack{a \\ t_{a}^{a}-t_{a}^{b}}} H\left(\theta_{a}^{\prime}, \theta_{b}^{\prime}\right) .
$$

In other words, this is the maximum value that the rectified output of a template $\theta_{a}$ will have if the input consists of only a signal $\theta_{b}$ having $S=1$. The role of templates and signals is, of course, interchangeable here. For $S \neq 1$, the maximum value will simply be $S \mathcal{H}$. We term this value the observed strength $S_{\text {obs }}$ of the signal.

Clearly, the larger the "width" of the intrinsic ambiguity function, the more sparsely can templates be placed around a signal in order to obtain the same detection probability. In this sense, the intrinsic ambiguity plays a central role in the determination of the density of templates and thus the computational cost of a one-step search. We first discuss, in the following section, the calculation of $\mathcal{H}$ and some of its relevant properties. This is followed by a discussion of template placement for a one-step search.

\section{A. Intrinsic ambiguity function}

Using the stationary phase Fourier transform given in Eq. (18), it can be shown easily that

$$
\begin{aligned}
H\left(\theta_{a}^{\prime}, \theta_{b}^{\prime}\right) & =\frac{1}{\beta}\left[F_{\cos }^{2}\left(\theta_{a}^{\prime}, \theta_{b}^{\prime}\right)+F_{\sin }^{2}\left(\theta_{a}^{\prime}, \theta_{b}^{\prime}\right)\right]^{1 / 2}, \\
F_{\cos }\left(\theta_{a}^{\prime}, \theta_{b}^{\prime}\right)= & \int_{f_{a}}^{f_{c}} \frac{d f}{S_{n}(f)} f^{-7 / 3} \cos [\alpha(f)], \\
F_{\sin }\left(\theta_{a}^{\prime}, \theta_{b}^{\prime}\right)= & -\int_{f_{a}}^{f_{c}} \frac{d f}{S_{n}(f)} f^{-7 / 3} \sin [\alpha(f)], \\
\alpha(f)= & 2 \pi\left[\Delta t_{a} f+\sum_{k} \Delta \tau_{k} \psi_{k}(f)\right] ; \\
& k \in[0,1,1.5], \\
\beta= & \int_{f_{a}}^{f_{c}} \frac{d f}{S_{n}(f)} f^{-7 / 3},
\end{aligned}
$$

where $\Delta t_{a}=t_{a}^{a}-t_{a}^{b}, \Delta \tau_{k}=\tau_{k}^{a}-\tau_{k}^{b}$. The quantities $F_{\text {cos }} / \beta$ and $F_{\sin } / \beta$ are nothing but the quantities $r$ and $s$ defined in Eq. (49) and Eq. (50) but expressed in terms of the stationary phase Fourier transform. They can, of course, be calculated exactly by generating the wave forms in time domain using Eq. (6) and computing their correlations using the FFT. We find that $F_{\cos }$ and $F_{\text {sin }}$ reproduce the corresponding exact quantities quite faithfully for both the initial and advanced LIGO noise spectral densities. This also holds to a large extent when $f_{c}$ is replaced by the least of the plunge cutoff frequencies corresponding to the two wave forms. Thus, $H\left(\theta_{a}^{\prime}, \theta_{b}^{\prime}\right)$ calculated using Eq. (72) also reproduces faithfully the corresponding exact results and this would also be true for $\mathcal{H}$ provided the location of the maximum in Eq. (71) is obtained accurately.

We obtain the location of the maximum in Eq. (71) in two steps. First, an initial estimate for the required value of $\Delta t_{a}$ is obtained as described below. This is followed by a search for the true maximum around this initial guess using a bracketing and golden search algorithm (MNBRAK and GOLDEN in [13]). In order to get the initial estimate, we solve the integrals in Eq. (73) and Eq. (74) using a stationary phase approximation but with the point of stationarity chosen in such a way as to yield the maximum value for the RHS in Eq. (72). Let the desired stationary point be $f=f_{0}$. Then, for fixed values of $\Delta \tau_{1.5}$ and $\Delta \tau_{0}$, the condition of stationarity yields

$$
\Delta t_{a}=-\left(\Delta \tau_{0}+\Delta \tau_{1}-\Delta \tau_{1.5}\right)+\Delta \tau_{0} x^{8}+\Delta \tau_{1} x^{6}-\Delta \tau_{1.5} x^{5},
$$

where $x=\left[f_{0} / f_{a}\right]^{-1 / 3}$. Substituting the above back into the integrands in Eq. (72) and maximizing the resulting expression over $f_{0}$ yields the required value. This value of $f_{0}$ is obtained only once for a particular choice of $\Delta \tau_{1.5}$ and $\Delta \tau_{0}$. For any other $\left(\Delta \tau_{1.5}, \Delta \tau_{0}\right)$, the same value of $f_{0}$ is used to obtain an initial estimate for $\Delta t_{a}$ using Eq. (77). The algorithm given above is quite fast as compared to the exact calculation. 


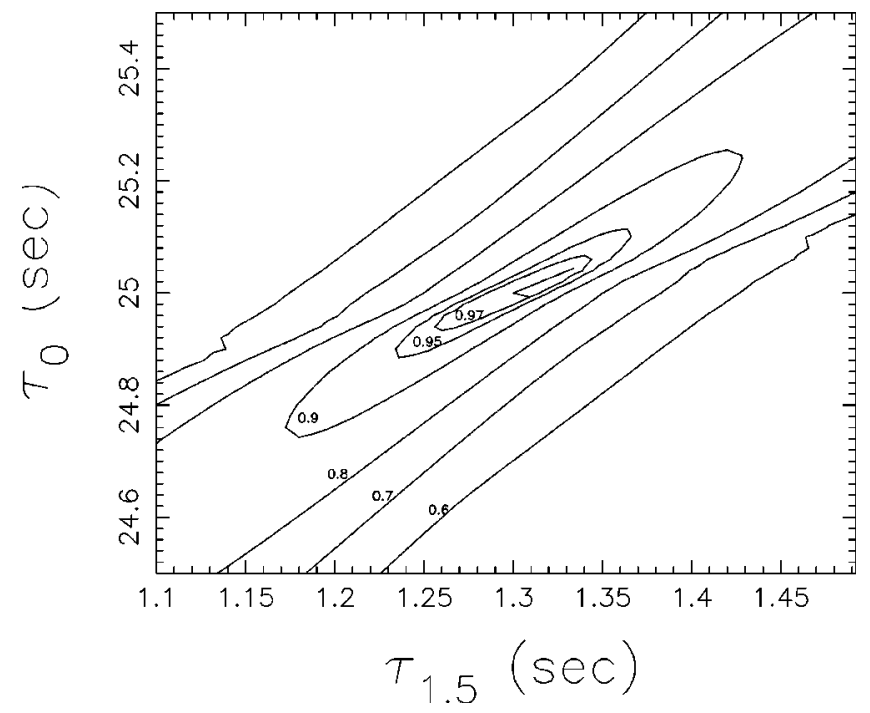

FIG. 4. The contours of the intrinsic ambiguity function $\mathcal{H}\left(\theta_{a}, \theta_{b}\right)$ for the initial LIGO. In this figure, $\theta_{a}$ is kept fixed at $\theta_{a}=(1.3,25.0) \mathrm{sec}$ and $\theta_{b}$ is varied. Also shown are the semi-minor and semi-major axes of the 0.97 contour as calculated from the Hessian $\mathbf{H}\left(\theta_{a}\right)$. The axes do not appear at a right angle to one another because the axes scales are different.

Contour plots of $\mathcal{H}\left(\theta_{a}, \theta_{b}\right)$, as a function of $\theta_{b}$ with $\theta_{a}$ fixed, are shown in Fig. 4. Also shown are the eigenvectors of the Hessian of $\mathcal{H}$ which is defined as

$$
\mathbf{H}_{i j}\left(\theta_{a}\right)=\left.\frac{1 \partial^{2} \mathcal{H}\left(\theta_{a}, \theta_{b}\right)}{2 \partial \theta_{b}^{i} \partial \theta_{b}^{j}}\right|_{\theta_{b}=\theta_{a}} .
$$

The Hessian $\mathbf{H}_{i j}\left(\theta_{a}\right)$ is identical to the metric on the signal manifold introduced in [4]. Since $\mathcal{H}\left(\theta_{a}, \theta_{a}+\Delta \theta\right)$ is maximum at $\Delta \theta=0$, the $\mathcal{H}$ surface is quadratic in a sufficiently small neighborhood of $\theta_{a}$ and, as shown in Fig. 4, the innermost contours are elliptical. The orientation and axis lengths of such an elliptical contour can be obtained in terms of the eigenvalues and eigenvectors of the Hessian. Let $\lambda_{1}\left(\theta_{a}\right)$, $\lambda_{2}\left(\theta_{a}\right)$ be the smaller and larger eigenvalues of $\mathbf{H}\left(\theta_{a}\right)$ respectively and let $\hat{e}_{1 a}$ and $\hat{e}_{2 a}$ be the corresponding eigenvectors (normalized to unity). Then, the length of the semiminor and semi-major axes of a contour at level $\epsilon$ are given by

$$
\begin{aligned}
& l_{1}\left(\epsilon, \theta_{a}\right)=\frac{\sqrt{1-\epsilon}}{\sqrt{-\lambda_{1}\left(\theta_{a}\right)}}, \\
& l_{2}\left(\epsilon, \theta_{a}\right)=\frac{\sqrt{1-\epsilon}}{\sqrt{-\lambda_{2}\left(\theta_{a}\right)}},
\end{aligned}
$$

respectively, while their orientation is given by the respective eigenvectors. Note that since the eigenvalues are negative, $\left|\lambda_{1}\left(\theta_{a}\right)\right| \geqslant\left|\lambda_{2}\left(\theta_{a}\right)\right|$. Since, we already have a nearly accurate method for computing $\mathcal{H}, \mathbf{H}_{i j}$ can be calculated simply by approximating the derivatives in Eq. (78) by finite differences. It should be noted that at a given location, the directions in which the contours at successively lower levels are most elongated suffer progressively larger rotations with
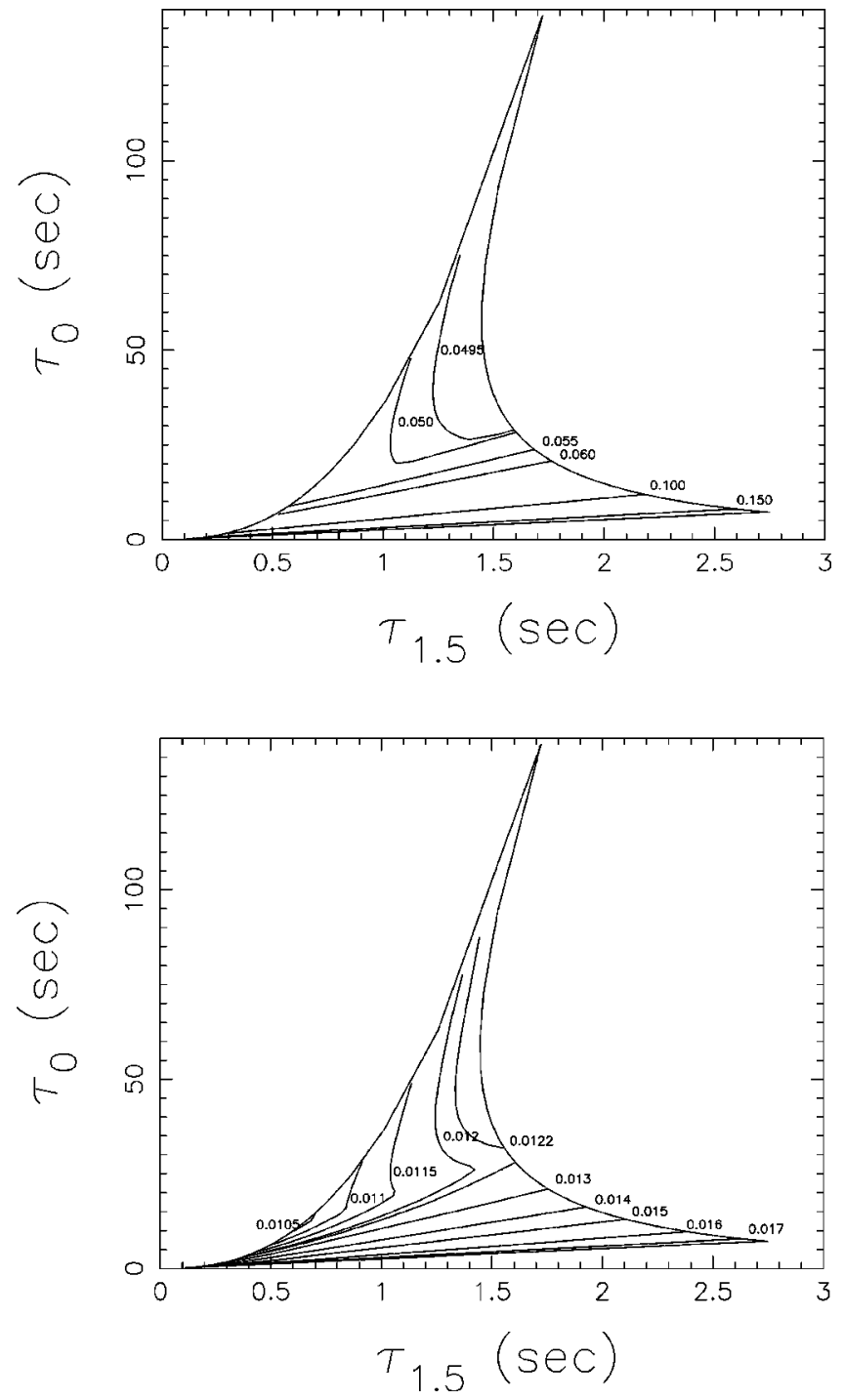

FIG. 5. The upper figure shows the contours of $l_{2}\left(0.97, \theta_{a}\right)$ and the lower figure shows the contours of $l_{1}\left(0.97, \theta_{a}\right)$, in the space of interest for the initial LIGO.

respect to these eigenvectors. We call this the shear of the contours. Typically, $\lambda_{i}\left(\theta_{a}\right)$ and $\hat{e}_{i a}$ provide a good estimate of the size and orientation of the contours for $\mathcal{H} \geqslant 0.95$.

The intrinsic ambiguity $\mathcal{H}$ is not independent of its location in parameter space. That is, if $\Delta \theta=\left(\Delta \tau_{1.5}, \Delta \tau_{0}\right)$ is a displacement vector, $\mathcal{H}\left(\theta_{a}, \theta_{a}+\Delta \theta\right) \neq \mathcal{H}\left(\theta_{b}, \theta_{b}+\Delta \theta\right)$ in general for $\theta_{a} \neq \theta_{b}$. For our purpose, the most appropriate way of characterizing this location dependence would be to investigate the change in the dimensions of the innermost contours of $\mathcal{H}$. This is because we are primarily interested in the detection of signals with low strengths and for such signals the templates in a one step search would be placed closely. For instance, we find from earlier works (MD96 and [4]) that for low values of $S$, the spacing of templates is such that the signal with the lowest detection probability has $\mathcal{H}\left(\theta_{t}, \theta_{s}\right) \simeq 0.97$, for some template $\theta_{t}$. In Figs. 5, 6 and Figs. 7,8 , we have plotted $l_{1}\left(0.97, \theta_{a}\right), l_{2}\left(0.97, \theta_{a}\right)$, the area of the ellipse $\pi l_{1} l_{2}$ and the angle between the $\tau_{1.5}$ axis and $\hat{e}_{1 a}$ as functions of $\theta_{a}$. The lowest contour level in each plot 

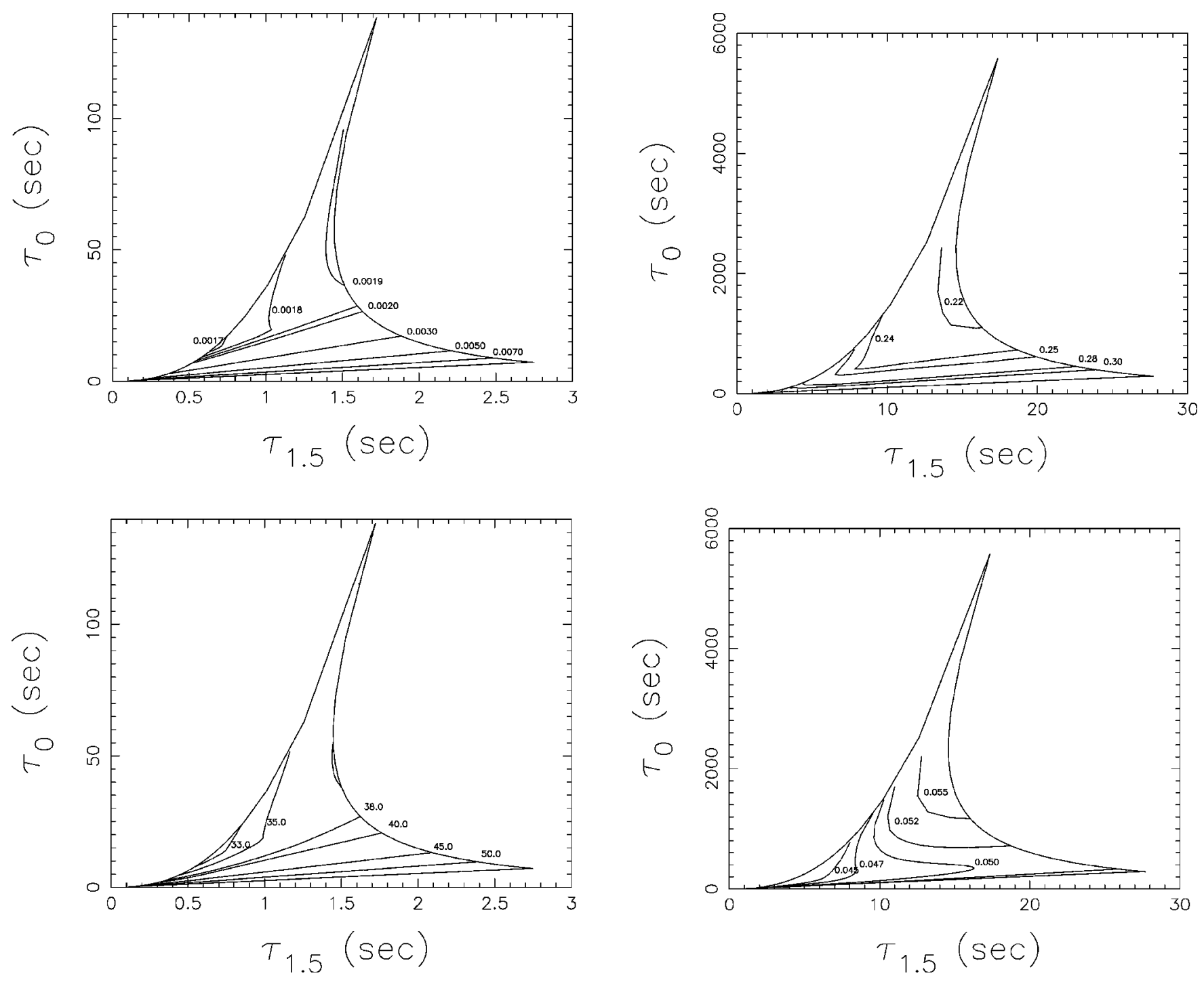

FIG. 6. The upper figure shows the area $\pi l_{1}\left(0.97, \theta_{a}\right) l_{2}\left(0.97, \theta_{a}\right)$ of the 0.97 contour of the intrinsic ambiguity function $\mathcal{H}$ and the lower figure shows the contours of $\alpha_{1}$, the angle (in degrees) between $\hat{e}_{1 a}$ and the $\tau_{1.5}$ axis, on the space of interest for the initial LIGO.

is close to the minimum value which that quantity takes over the whole of the space of interest.

\section{B. Geometrical configuration of a one-step template bank}

In MD96, we had introduced a set of criteria which a template bank for a one-step search was required to satisfy. These criteria were (C1) every signal, having a strength $S$ greater than a given minimum strength $S_{\min }$, should have a detection probability greater than a given minimum detection probability $Q_{\mathrm{d}, \min }$ and (C2) the false alarm should stay below a specified level $Q_{0, \max }$. Throughout the following, $Q_{\mathrm{d} \text {,min }}$ $=0.95$ and $Q_{0, \text { max }}$ is chosen to be such that the average rate of false events is 1 event/yr. Apart from the above criteria, we also demand (C3) that the templates be spaced as sparsely as possible so as to minimize the computational cost.

An obvious way to fix the template placement would be to search through all the possible placement configurations and find the one satisfying the three criteria stated above. We

FIG. 7. The upper figure shows the contours of $l_{2}\left(0.97, \theta_{a}\right)$ and the lower figure shows the contours of $l_{1}\left(0.97, \theta_{a}\right)$, in the space of interest for the advanced LIGO.

have already introduced formulas for detection and false alarm probabilities in Sec. III which can be used in checking (C1) and (C2). However, such a blind search in configuration space is impossible to perform in practice since the number of templates can be expected to be quite large. Instead one can make some reasonable assumptions regarding the geometry of the final configuration and then proceed to perform a limited search within that framework. We will now present an argument that suggests an approximation to the optimum geometry of template placement in the post ${ }^{1.5}$-Newtonian case. This approximation should be good enough for estimating the performance of a two-step hierarchical search but a more careful analysis would be required when such a scheme is actually implemented.

\section{Case of location independent $\mathcal{H}$}

Consider, first, a simple hypothetical situation in which the intrinsic ambiguity is location independent, that is, $\mathcal{H}\left(\theta_{a}, \theta_{a}+\Delta \theta\right)=\mathcal{H}(\Delta \theta)$. Recall that the detection probability of a signal was determined by a subset $\mathcal{Z}$ of samples belonging to the rectified outputs of templates in some neigh- 

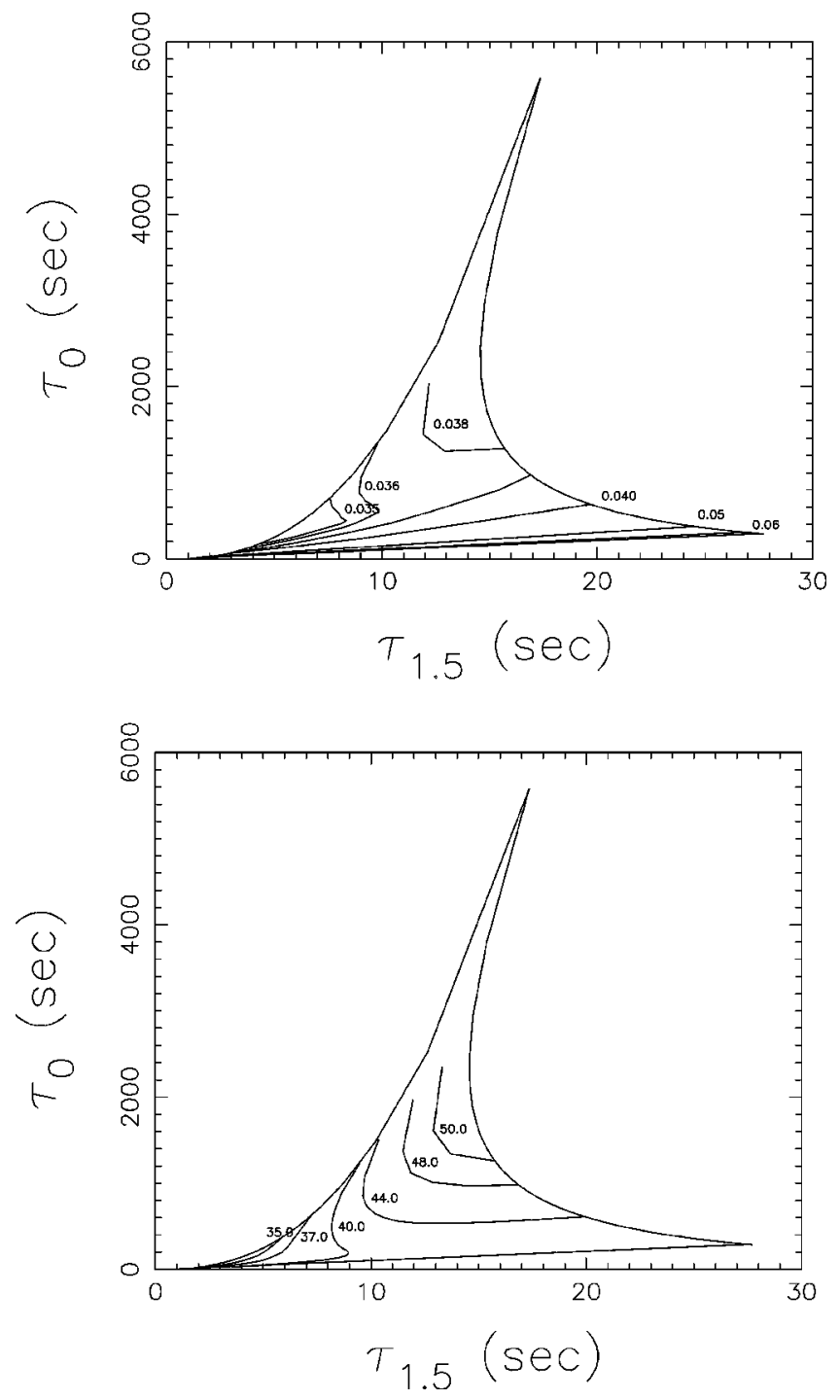

FIG. 8. The upper figure shows the area $\pi l_{1}\left(0.97, \theta_{a}\right) l_{2}\left(0.97, \theta_{a}\right)$ of the 0.97 contour of the intrinsic ambiguity function $\mathcal{H}$ and the lower figure shows contours of $\alpha_{1}$, the angle (in degrees) between $\hat{e}_{1 a}$ and the $\tau_{1.5}$ axis, in the space of interest for the advanced LIGO.

borhood of the signal. Thus, the detection probability of a signal is determined entirely by the local distribution of templates around it. Let the coordinates of the signal be $\theta_{s}$ and that of the templates be $\left\{\theta_{s}+\Delta \theta_{1}, \ldots, \theta_{s}+\Delta \theta_{p}\right\}$, where $P$ is the number of templates in the neighborhood. Also, the samples in $\mathcal{Z}$ which contribute most to the detection probability are the ones which are located at the maxima of the rectified outputs (for zero noise) of these templates. Let the set of such samples be $\mathcal{Z}^{\prime}=\left\{\mathcal{Z}_{i}^{\prime}\right\} \subset \mathcal{Z}, i=1, \ldots, P$. The remaining samples typically contribute only a few percent more to the detection probability. Then,

$$
\overline{\mathcal{Z}_{i}^{\prime}} \simeq S \mathcal{H}\left(\theta_{s}, \theta_{s}+\Delta \theta_{i}\right) .
$$

Now, if $\mathcal{H}$ is assumed to be location independent, two different signals (having the same strength) would have the same detection probability provided the local configuration of templates $\left\{\Delta \theta_{1}, \ldots, \Delta \theta_{p}\right\}$ around them is the same. Strictly speaking, this statement is not true unless $H\left(\theta_{a}^{\prime}, \theta_{b}^{\prime}\right)$

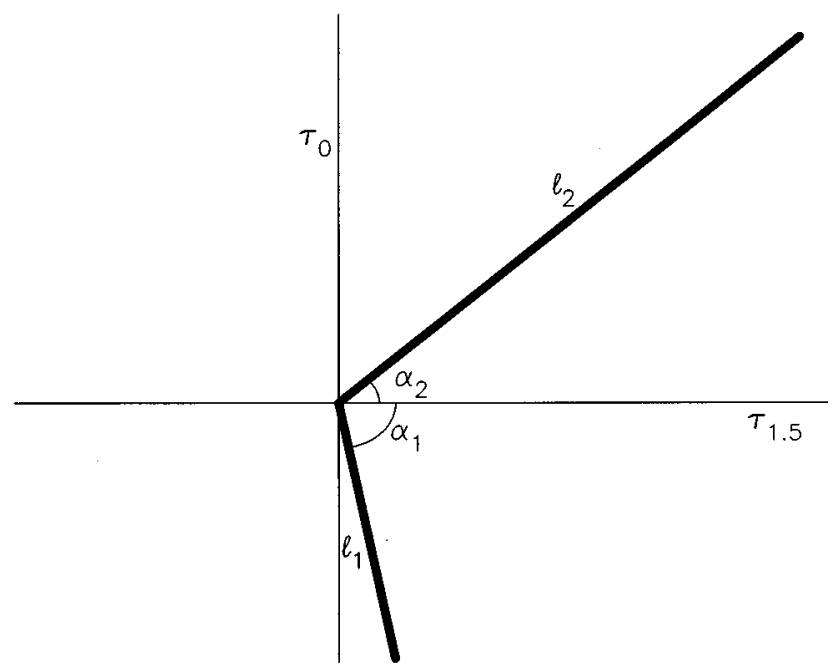

FIG. 9. The parameters of a unit cell which is a parallelogram. Whenever the sides of the unit cell are assumed to be along the eigenvectors of the Hessian, $l_{1}$ is taken to be along the semi-minor axis while $l_{2}$ is taken along the semi-major axis.

is also independent of location. This is because the covariance matrix (determined by $H$ ) of the set of samples $\mathcal{Z}^{\prime}$ need not be location independent even though their mean values (given by $\mathcal{H}$ ) may be so. However, we will proceed with the assumption that variation in the covariance matrix is a negligible effect. The validity of this assumption can be checked after the final results have been obtained, as we do below.

An intrinsic ambiguity function which is location independent is not unrealistic since the Newtonian $[6,12]$ as well as the post ${ }^{1}$-Newtonian [4] wave forms are known to have such an intrinsic ambiguity for the right choice of parameters. In fact, in these cases, the function $H\left(\theta_{1}^{\prime}, \theta_{2}^{\prime}\right)$ itself is dependent only on $\Delta \theta$. Thus, the detection probability of a signal in the case of Newtonian or post ${ }^{1}$-Newtonian wave forms depends strictly on the local configuration $\left\{\Delta \theta_{1}, \ldots, \Delta \theta_{p}\right\}$ alone.

Now consider a configuration of templates in which the distribution of templates in the space of interest is inhomogeneous but which is claimed to satisfy (C1), (C2), and (C3) for some $S_{\min }$. It then follows that the detection probability of a signal with $S=S_{\min }$ in a region sparsely populated by templates is at least $Q_{\mathrm{d}, \min }$. However, this implies that a region where templates are spaced densely is overpopulated because, as shown above, the same sparse local distribution should suffice everywhere. Therefore, a further reduction in computational cost can be brought about by removing some of the templates from the overpopulated region. This implies that an inhomogeneous distribution cannot be an optimal one. Hence, when $\mathcal{H}$ is location independent, templates should be distributed homogeneously which is equivalent to placing them on a regular grid.

A two dimensional regular grid is specified by a single unit cell. In the present paper we will assume that this unit cell is a parallelogram. Such a unit cell is specified by the lengths of two adjacent sides, $l_{1}$ and $l_{2}$, and the angles, $\alpha_{1}$, $\alpha_{2}$, that $l_{1}$ and $l_{2}$ make with some reference direction (see Fig. 9). To find the optimal placement of templates, therefore, a search can be performed in $\left(l_{1}, l_{2}, \alpha_{1}, \alpha_{2}\right)$ space for the unit cell having the largest area under the constraint that 
the resulting grid of templates satisfy $(\mathrm{C} 1)$ and $(\mathrm{C} 2)$. Such a search would be computationally expensive even with the fast methods that were introduced in the earlier sections. Note, however, that the unit cell with the largest area, for a given $l_{1}$ and $l_{2}$, is a rectangle. Hence, for a given $l_{1}$ and $l_{2}$, a search should first be performed among all rectangular unit cells to locate the ones that satisfy $(\mathrm{C} 1)$ and $(\mathrm{C} 2)$. This is equivalent to just a rotation of the grid which involves only one of the angles and, hence, saves significantly on computations. Once the largest rectangular unit cell that satisfies (C1) and (C2) has been found, the search can then be extended to non-rectangular unit cells with larger areas.

The computational cost can be reduced further by making an educated guess for the orientation of the rectangular unit cell. For instance, let the contours of $\mathcal{H}$ be ellipsoids with the same orientation. Then it can be seen heuristically that the largest rectangular unit cell should be obtained when $l_{1}$ and $l_{2}$ are oriented along the major and minor axes, that is, the eigenvectors of the Hessian $\mathbf{H}$. If the contours exhibit a shear, then the largest rectangular unit cells for different values of $S_{\min }$ would, of course, be oriented differently. In such a case also, the computation involved in the search can be reduced by starting with an orientation given by the eigenvectors of $\mathbf{H}$ and then searching a small range of $\alpha_{1}$ around this orientation.

In order to check that the above argument is reasonable, consider Fig. 10. For a rectangular unit cell oriented along the $\tau_{1.5}$ and $\tau_{0}$ axes and having arbitrary dimensions $l_{1}$ and $l_{2}$, we show the detection probability of signals, having the same strength, which lie in the interior of the cell. The threshold has also been chosen arbitrarily and samples for the set $\mathcal{Z}$ were chosen from the rectified outputs of the four templates at the vertices of the unit cell. Also superimposed on this detection probability map are the contours of $\mathcal{H}$ for one of the templates. It is clear from the figure that, as expected, the detection probability map closely follows the contours of the intrinsic ambiguity. Roughly speaking, the detection probability contours are formed by the "overlap" of the $\mathcal{H}$ contours. Therefore, it can be expected that if the unit cell were oriented along the eigenvectors of $\mathbf{H}$, then the area can be made larger, keeping the minimum detection probability the same, because this orientation would maximize the overlap of the $\mathcal{H}$ contours. In Fig. 10 we also show the detection probability map, for the same threshold as above, when the unit cell is oriented along the eigenvectors of $\mathbf{H}\left(l_{1}\right.$ along $\hat{e}_{1 a}$ and $l_{2}$ along $\left.\hat{e}_{2 a}\right)$. The minimum detection probability is much larger now which implies that the area can now be increased further. In the following, we will restrict the parameter space for the unit cell to be only $\left(l_{1}, l_{2}\right)$ and orient the sides along the eigenvectors of the Hessian. That is, the sides of the unit cell are given by $l_{1} \hat{e}_{1 a}$ and $l_{2} \hat{e}_{2 a}$.

\section{Case of post ${ }^{1.5}$-Newtonian $\mathcal{H}$}

Consider the family of post ${ }^{1.5}$-Newtonian wave forms now. As shown in the previous section, $\mathcal{H}$ is not location independent in this case. However, if this variation is small over the scale of few unit cells, then it can be expected that the optimum template placement for the post ${ }^{1.5}$-Newtonian case would also be close to a regular grid. In earlier works
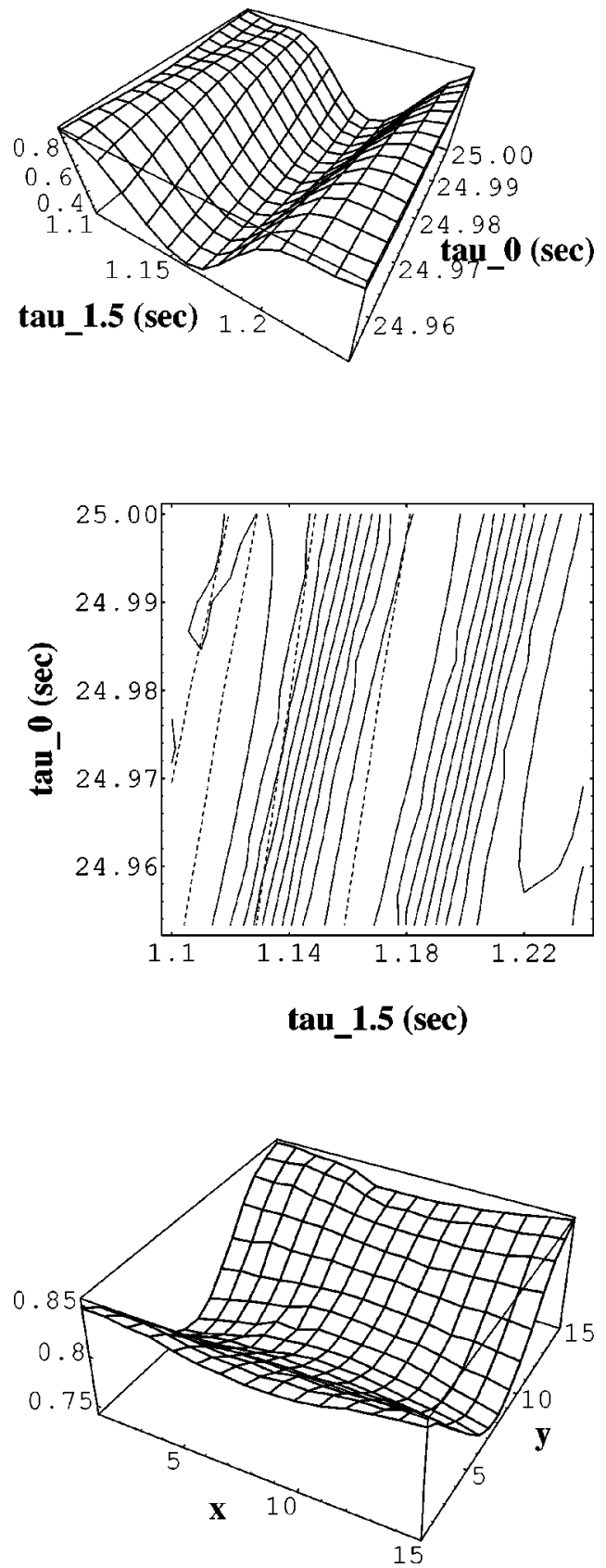

FIG. 10. In the uppermost figure we show the detection probability of signals in the interior of a rectangular unit cell which is oriented along the $\tau_{1.5}$ and $\tau_{0}$ axes with the top left vertex at $(1.1,25.0) \mathrm{sec}$. The length $l_{1}$ of the side along the $\tau_{0}$ axis is $0.05 \mathrm{sec}$ while the length $l_{2}$ of the other side is $0.150 \mathrm{sec}$. The threshold and signal strengths were chosen (arbitrarily) as $\eta=8.0$ and $S=9.0$. In the middle figure the contours of this detection probability map are superimposed on some of the contours (dashed line) of $\mathcal{H}\left(\theta_{a}, \theta_{b}\right)$ with $\theta_{a}=(1.1,25.0) \mathrm{sec}$. In the lowermost figure, we show the detection probability map for the same unit cell but now with $l_{1}$ and $l_{2}$ oriented along the eigenvectors $\hat{e}_{1 a}$ and $\hat{e}_{2 a}$. The threshold and signal strength are the same as in the figures above it. The values along the $x$ axis (oriented along $\hat{e}_{1 a}$ ) and $y$ axis (oriented along $\hat{e}_{2 a}$ ) are the serial numbers of the grid points.

(MD96 and [4]), the typical spacing of templates for low values of $S_{\min }$ turned out to be such that for any signal, the value of $\mathcal{H}$ was at least $\sim 0.97$ in some template. In the present case, if templates are placed along the eigenvectors 

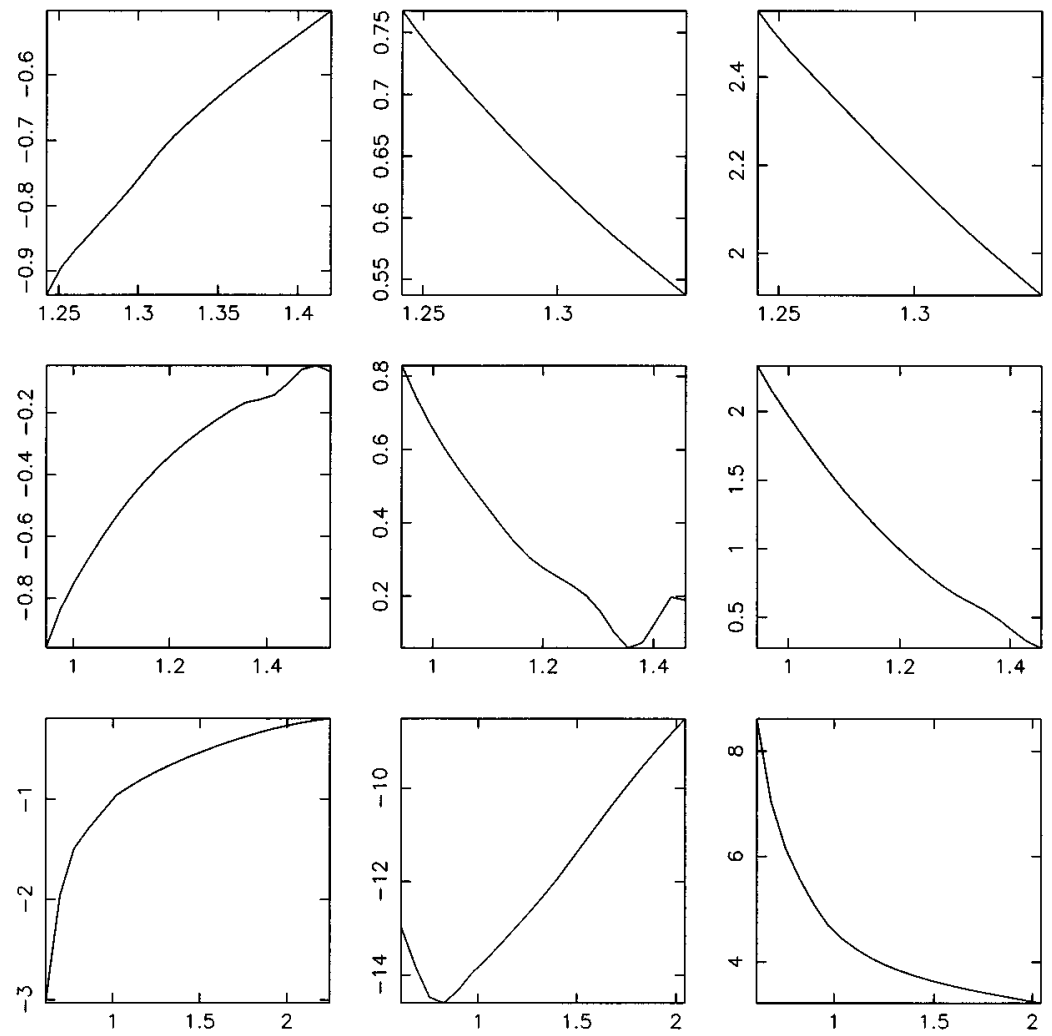

FIG. 11. The quantities $\delta_{1}, \delta_{2}$ and $\delta_{\alpha}$ [see Eqs. (82) and (83)] for the case of the initial LIGO. Each row of figures corresponds to a fixed value of $\tau_{0}$. (i) For the first row, $\tau_{0}=60.0 \mathrm{sec}$, (ii) for the second row, $\tau_{0}=30.0 \mathrm{sec}$, and (iii) for the third row, $\tau_{0}=10.0 \mathrm{sec}$. The first column corresponds to $\delta_{1}$, the second corresponds to $\delta_{2}$ and the third to $\delta_{\alpha}$. The $x$ axis is the $\tau_{1.5}$ axis.

of the Hessian, this would imply that the typical lengths for the sides of the unit cell are $\sim 2 l_{1}\left(0.97, \theta_{a}\right)$ and $\sim 2 l_{2}\left(0.97, \theta_{a}\right)$. Thus, the effect of the location dependence of $\mathcal{H}$ on the placement of templates can be studied by comparing the change in $l_{i}$, over a distance $2 l_{i}$ along $\hat{e}_{i a}$ [that is, $\left.l_{i}\left(0.97, \theta_{a}+\hat{e}_{i a}\right)-l_{i}\left(0.97, \theta_{a}\right)\right]$, with the value of $l_{i}$ at that point. For the scales used in Fig. 5 and Fig. 7, a line along any of the two eigenvectors would be nearly horizontal at any point. Therefore, the change in $l_{i}$ along $\hat{e}_{i a}$ can be obtained approximately by simply measuring the change over a line of constant $\tau_{0}$. In Figs. 11, 12, we plot the quantity

$$
\delta_{i}\left(\tau_{1.5} ; \tau_{0}^{0}\right)=\left[1-\frac{l_{i}\left[0.97,\left(\tau_{0}^{0}, \tau_{1.5}+2 l_{i}^{0}\right)\right]}{l_{i}\left[0.97,\left(\tau_{0}^{0}, \tau_{1.5}\right)\right]}\right] \times 100,
$$

where $l_{i}^{0}$ is the value of $l_{i}$ at the intersection of the $\tau_{0}=\tau_{0}^{0}$ line and the boundary $A B$ or $B C$ as the case may be [23]. A similar quantity $\delta_{\alpha}$ can be constructed for the angle $\alpha_{1}$ that the semi-minor axis makes with the $\tau_{1.5}$ axis,

$$
\delta_{\alpha}=\left[1-\frac{\alpha_{1}\left(\tau_{0}^{0}, \tau_{1.5}+2 l_{2}^{0}\right)}{\alpha_{1}\left(\tau_{0}^{0}, \tau_{1.5}\right)}\right] \times 100 .
$$

This has also been plotted in Figs. 11, 12.

It is evident that the variation of the dimensions and orientations of the unit cells, over the scale of a single unit cell itself, is quite small over a large portion of the space of interest for both the initial and advanced LIGO. In general, this variation becomes more rapid towards the high mass, or low $\tau_{0}$, region. However, it is still small for the advanced LIGO though it may have some significant effect in the case of the initial LIGO. Thus, at least in the case of the advanced LIGO, one can expect that the optimum placement of templates will be along an "adiabatically" changing grid over most of the space of interest. Since the rate of detectable events is not expected to be large for the initial LIGO, we will not investigate the placement of templates for the initial LIGO any further here. Instead we will concentrate on the advanced LIGO and assume that a quasi-regular grid will be obtained for the initial LIGO also. Further, in the following analysis, we will approximate the quasi-regular grid above by a set of piecewise regular grids, that is, a set of patches covering the whole of the space of interest where the unit cells in each patch are identical but differ in dimension and orientation in different patches.

Though, in principle, the unit cell in each patch can be determined by using the algorithm given earlier, this would again be impractical because now a placement algorithm would have to search a two dimensional parameter space, $l_{1}$ and $l_{2}$, for each patch. However, if the assumption that the detection probability is almost completely determined by $\mathcal{H}$ alone were true, then the search would collapse to just two dimensions. This can be seen as follows (we call this assumption As1 for convenience in the following). For a small displacement $\Delta \theta=x_{1} \hat{e}_{1 a}+x_{2} \hat{e}_{2 a}$ at $\theta_{a}$,

$$
\mathcal{H}\left(\theta_{a}, \theta_{a}+\Delta \theta\right) \simeq 1-\left[\lambda_{1}\left(\theta_{a}\right) x_{1}^{2}+\lambda_{2}\left(\theta_{a}\right) x_{2}^{2}\right]
$$

where we have rotated the coordinate system locally so that 

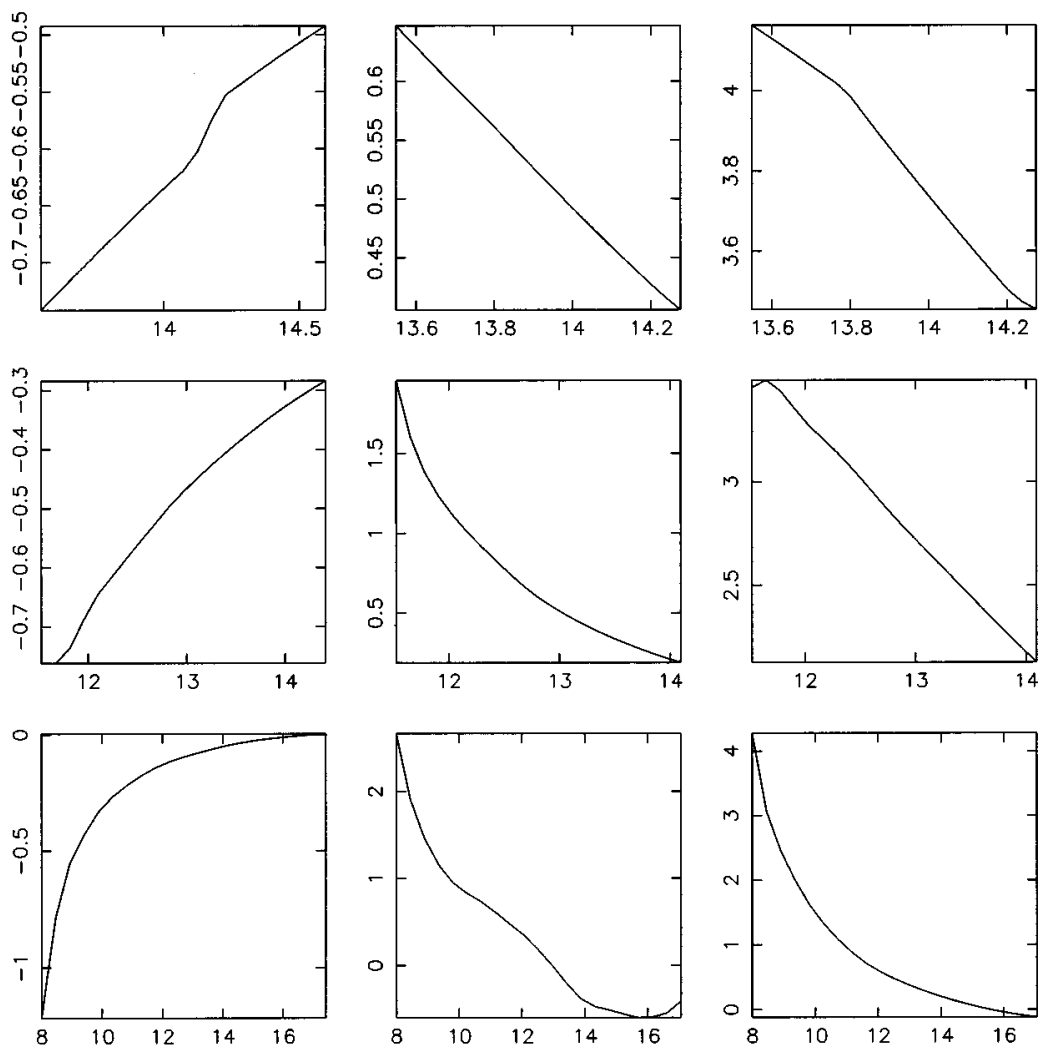

FIG. 12. The quantities $\delta_{1}, \delta_{2}$ and $\delta_{\alpha}$ [see Eqs. (63) and (64)] for the case of the advanced LIGO. Each row of figures corresponds to a fixed value of $\tau_{0}$. (i) For the first row, $\tau_{0}=3000.0 \mathrm{sec}$, (ii) for the second row, $\tau_{0}=2000.0 \mathrm{sec}$, and (iii) for the third row, $\tau_{0}=800.0 \mathrm{sec}$. The first column corresponds to $\delta_{1}$, the second corresponds to $\delta_{2}$ and the third to $\delta_{\alpha}$. The abscissa is the $\tau_{1.5}$ axis (in seconds).

$\mathbf{H}\left(\theta_{a}\right)$ is diagonalized. Consider a different location $\theta_{b}$ where $\lambda_{i}\left(\theta_{b}\right)=\alpha_{i} \lambda_{i}\left(\theta_{a}\right)$. Then, for a small displacement at $\theta_{b}$ [in a rotated coordinate system that diagonalizes $\left.\mathbf{H}\left(\theta_{b}\right)\right]$ and at the same order of approximation as in Eq. (84),

$$
\mathcal{H}\left[\theta_{b}, \theta_{b}+\sum x_{i} \hat{e}_{i b} / \sqrt{\alpha_{i}}\right]=\mathcal{H}\left[\theta_{a}, \theta_{a}+\sum x_{i} \hat{e}_{i a}\right]
$$

Now, suppose that the unit cell at $\theta_{a}$, having dimensions $l_{1}$ and $l_{2}$, satisfies $(\mathrm{C} 1)$ for a given threshold. This implies that every signal in the interior of the cell has a detection probability greater than $Q_{d \text {, min }}$. Let the relative coordinates of such a signal be $\theta_{s}=\left(\epsilon_{1} l_{1}, \epsilon_{2} l_{2}\right)$, where $0<\epsilon_{i}<1$. If As1 is true, then the detection probability must depend only on $\mathcal{H}_{1}=\mathcal{H}\left(\theta_{a}+\theta_{s}, \theta_{a}\right), \quad \mathcal{H}_{2}=\mathcal{H}\left(\theta_{a}+\theta_{s}, \theta_{a}+l_{1} \hat{e}_{1 a}\right), \quad \mathcal{H}_{3}$ $=\mathcal{H}\left(\theta_{a}+\theta_{s}, \theta_{a}+l_{2} \hat{e}_{1 a}\right) \quad$ and $\quad \mathcal{H}_{4}=\mathcal{H}\left(\theta_{a}+\theta_{s}, \theta_{a}+l_{1} \hat{e}_{1 a}\right.$ $+l_{2} \hat{e}_{2 a}$ ) (we have neglected the contribution from other templates for the present but this does not affect the argument). It then follows from Eq. (85) that, for the same threshold, the detection probability of a signal with relative coordinates $\left(\epsilon_{1} l_{1} / \sqrt{\alpha_{1}}, \epsilon_{2} l_{2} / \sqrt{\alpha_{2}}\right)$ at $\theta_{b}$ will be the same as that of $\theta_{s}$ at $\theta_{a}$. Hence, for a given threshold, if a unit cell with sides $l_{1}$, $l_{2}$ at $\theta_{a}$ satisfies $(\mathrm{C} 1)$, then so would a unit cell with sides $l_{1} / \sqrt{\alpha_{1}}, l_{2} / \sqrt{\alpha_{2}}$ at $\theta_{b}$.

Assume that for a given threshold the largest unit cell that is compatible with $(\mathrm{C} 1)$ is unique (note that the orientation of unit cells has already been fixed and only rectangular unit cells are being considered). We call this assumption As2.
Now, suppose that the optimum solution compatible with all the three criteria (C1), (C2), and (C3) has been obtained by some means. That is, the sides of the largest unit cell compatible with $(\mathrm{C} 1)$ in each patch as well as a common threshold have been found. From As2 and As1, it then follows that if the unit cell dimensions are $l_{1}$ and $l_{2}$ in any one patch, the dimensions of a unit cell in any other patch must be $l_{1} / \sqrt{\alpha_{1}}$ and $l_{2} / \sqrt{\alpha_{2}}$. Hence, when searching the $2 N_{p}$ parameter space of unit cell dimensions $\left(N_{p}\right.$ being the number of patches), only the subspace $l_{1}, l_{2}$ for any one unit cell needs to be searched. We should emphasize here that the above argument is by no means a rigorous proof. Given the complicated interdependences of various quantities (for instance, even the number of patches and also the extent of a patch may depend on the dimensions of the unit cells), it would be difficult to cast the problem into a tractable mathematical form. However, we find the above argument sufficiently suggestive and the conclusions reached as plausible. The assumption As2 is actually not required since it can be expected that the total number of templates, hence the threshold for a given false alarm, will only depend on the areas of the unit cells in each patch and not on their individual dimensions. Thus, one can always choose the optimum solution to be the one where unit cells are scaled verisons of each other, without violating (C2) or (C3).

How large can $l_{1}$ and $l_{2}$ be in the post ${ }^{1.5}$-Newtonian case before the detection probability for scaled unit cells starts showing significant errors? We have checked this empirically and the results are presented in Figs. 13 (initial LIGO) and 14 (advanced LIGO). In each figure we present our re- 

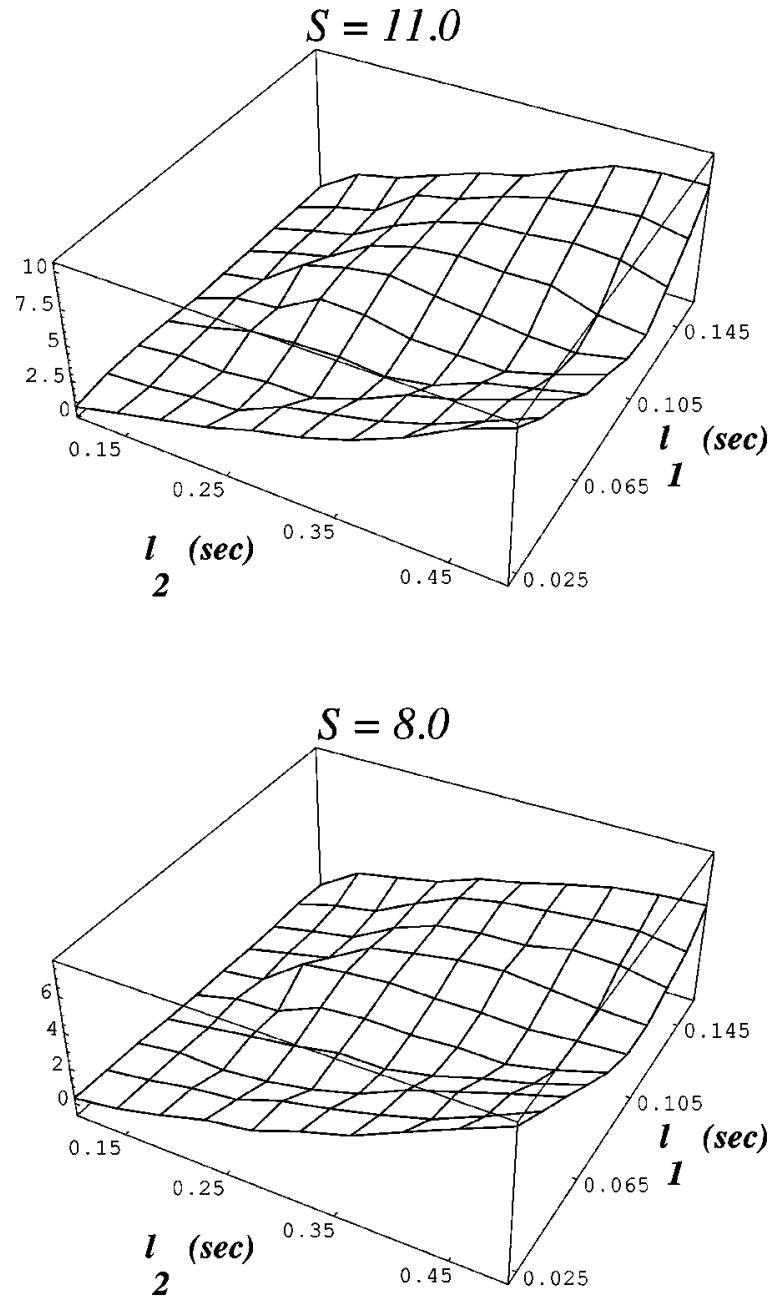

FIG. 13. The relative error in detection probability for corresponding signals in two widely separated unit cells. Let the locations of the unit cells be $\theta_{a}$ and $\theta_{b}$. In this figure, we consider the case of the initial LIGO and place the top left templates of the unit cells at $\theta_{a}=(1.3,50.0) \mathrm{sec}$ and $\theta_{b}=(1.5,10.0) \mathrm{sec}$. The value used for the signal strength $S$ is shown at the top of each plot. The effect of plunge cutoff has been incorporated in the calculations.

sults for different values of $S_{\min }$ which are chosen to encompass the typical range of $S_{\min }$ that will be considered later. The detection probability that we consider throughout our analysis is 0.95 . Hence, we compute the errors in detection probability at the 0.95 level. Also, it is not enough to compute the error for only one signal point since it may depend on the signal location. Therefore, the maximum error among three different signals is shown.

For each plot, we take two widely separated locations $\theta_{A}$ and $\theta_{B}$. The $x$ axis and $y$ axis are the values of $l_{1}$ and $l_{2}$ at $\theta_{A}$. The corresponding quantities at $\theta_{B}$ are $l_{1}^{\prime}=l_{1} / \sqrt{\gamma_{1}}$ and $l_{2}^{\prime}=l_{2} / \sqrt{\gamma_{2}}$, where $\gamma_{i}=\lambda_{i}\left(\theta_{B}\right) / \lambda_{i}\left(\theta_{A}\right)$. At each location the detection probabilities of three representative signals are obtained (this anticipates the discussion of the Sec. IV D), namely, the signals $\theta_{1 a}=\theta_{a}+\left(l_{1} \hat{e}_{1 a}+l_{2} \hat{e}_{2 a}\right) / 2, \quad \theta_{2 a}=\theta_{a}$ $+l_{1} \hat{e}_{1 a} / 2$ and $\theta_{3 a}=\theta_{a}+l_{2} \hat{e}_{2 a} / 2$, where $a=A$ or $B$. Let the threshold at which the detection probability of $\theta_{i A}$ equals 0.95 be $\eta_{i}$. Let the detection probability of $\theta_{i B}$ at the same threshold $\eta_{i}$ be $Q_{d i}$. The quantity plotted on the $z$ axis is the maximum relative error among the three signals. That is,
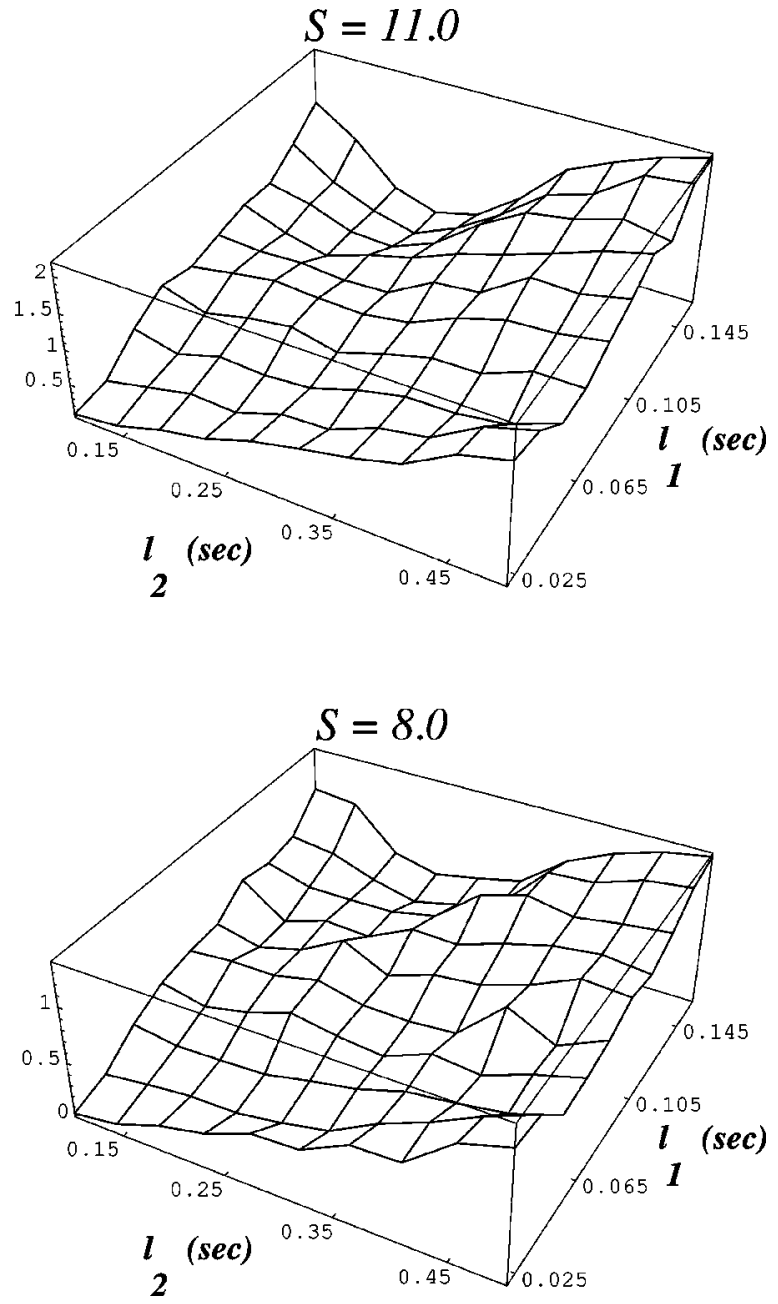

FIG. 14. The relative error in detection probability for corresponding signals in two widely separated unit cells. Let the locations of the unit cells be $\theta_{a}$ and $\theta_{b}$. In this figure, we consider the case of the advanced LIGO and place the top left templates of the unit cells at $\theta_{a}=(13.0,2000.0) \mathrm{sec}$ and $\theta_{b}=(15.0,400.0) \mathrm{sec}$. The value used for the signal strength $S$ is shown at the top of each plot. The effect of plunge cutoff has been incorporated in the calculations.

$$
\max _{i}\left[1-\frac{Q_{d i}}{0.95}\right] \times 100
$$

Thus, we are plotting the maximum relative error in the detection probability (at the 0.95 level) as a function of the unit cell dimensions. As mentioned earlier, the typical one-step spacings that can be expected are $2 l_{1}\left(0.97, \theta_{a}\right)$ and $2 l_{2}\left(0.97, \theta_{a}\right)$. For these values, we see from the figures that the typical error is $\leqslant 2 \%$. In fact, the errors stay small for much larger values of the unit cell dimensions. Hence, for a one-step template placement involving low values of $S_{\min }$, As 1 can be assumed to be valid for post ${ }^{1.5}$-Newtonian wave forms.

\section{Number of templates for a one-step search for post ${ }^{1.5}$-Newtonian wave forms}

In order to obtain the computational cost of a one-step search as well as the threshold for a given false alarm, the 


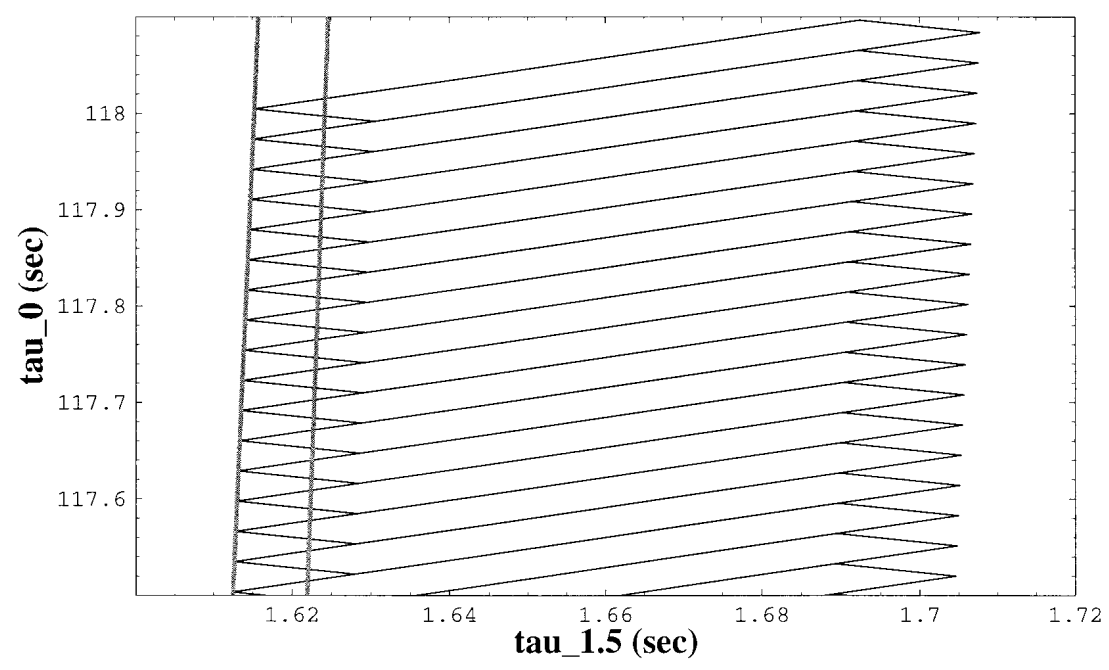

FIG. 15. A schematic illustration of a quasi-regular grid of unit cells near the vertex $A$ of the space of interest (initial LIGO). The lengths used for the sides of the unit cells are $l_{1}=0.02 \mathrm{sec}$ and $l_{2}=0.120 \mathrm{sec}$. These lengths have been chosen arbitrarily but represent typical values obtained in a one-step search. The boundary of the space of interest is shown by the lighter lines. The top left corner of each unit cell is placed on the left most boundary which is the image of the principal diagonal in the $\left(m_{1}, m_{2}\right)$ plane.

number of templates in a grid has to be obtained. This is not a straightforward task, however, because of the non-trivial shape of the boundary and the variation in the area of the contours with a change in location.

The non-trivial shape of the boundary would cause some templates in any regular grid to fall outside the space of interest. However, as can be seen from Figs. 1 and 2, in which the unit cells are almost horizontal because of the scales used, such an effect would be significant only near the region around vertex $A$. In this region, even a single unit cell may span both the boundary segments $A C$ and $A B$. Note, however, that the segments $A B$, as well as $B C$, are not strict limits. That is, astrophysically valid templates can also exist beyond them. This is not true, however, for the segment $A C$ which is the image of the principal diagonal in the $\left(m_{1}, m_{2}\right)$ plane. Thus, although no template should be placed on the left of $A C$, it is acceptable if some templates in a grid breach $A B$ or $B C$. This allows a quasi-regular grid to be placed in the region near $A$, as shown schematically in Fig. 15. This patch of templates can be used to cover the space of interest from $A$ until that value of $\tau_{0}$ at which the width of the space of interest becomes comparable to the length $l_{2}$ of the unit cell.

From Fig. 15, it appears that it may not be necessary to include the templates on the right hand edges of the unit cells. However, these templates would be required in the region where the size of the unit cell becomes comparable to the width of the space of interest, in which case the signal at the centroid of the unit cells (which needs the right hand templates for achieving the required detection probability) would lie inside the space of interest. For simplicity in the counting algorithm we include the right hand templates for unit cells which lie higher up (near vertex A) also. The number of extra templates thus added will not be significant compared to the total number of templates that will be required to cover all of the space of interest and, hence, will not significantly alter the final results.
The effect of the variation in the area of the $\mathcal{H}$ contours on the number of templates can be incorporated approximately as follows. Recall that in the previous section we showed that unit cells in different patches can be taken as scaled versions of a standard unit cell, where the scale factors were the ratio of the corresponding eigenvalues of the Hessian. Thus, the area of a unit cell will vary with location according to these scale factors only and, hence, the relative change in the area will be the same as that in the area of the 0.97 contour which is shown in Figs. 6 and 8.

Let $l_{1}$ and $l_{2}$ be the dimensions of the unit cell in that region of the space of interest where the variation in the area is small (say, maximum relative change of $\sim 15 \%$ ). For the initial LIGO this is roughly the region between vertex $A$ and the contour at $0.002 \mathrm{sec}^{2}$ in Fig. 6, while it is the area between $A$ and the contour at $0.04 \mathrm{sec}^{2}$ for the case of the advanced LIGO. Let $A_{C_{1}-C_{2}}$ be the area between contours $c_{1}$ and $c_{2}$ and $a=l_{1} l_{2}$. Then, for the case of initial LIGO, the number of templates that lie in $A_{0.002-0.003}$ would be approximately $A_{0.002-0.003} /(1.5 a)$ since the area of the contour increases by $\sim 50 \%$ in this region. Similarly, the number of templates in $A_{0.003-0.004}$ would be $\sim A_{0.003-0.004} /(2.0 a)$ and so on. Let $\beta_{C_{1}-C_{2}}=A_{C_{1}-C_{2}} / A$, where $A$ is the area of the whole of the space of interest [see Eq. (14)] and $N_{T}^{v}$ be the number of templates in the region where the variation in area is fast (i.e., below the $0.002 \mathrm{sec}^{2}$ contour). Then,

$$
\begin{aligned}
N_{T}^{v} \simeq & {\left[\frac{\beta_{0.002-0.003}}{1.5}+\frac{\beta_{0.003-0.004}}{2.0}+\frac{\beta_{0.004-0.005}}{2.5}+\frac{\beta_{0.005-0.006}}{3.0}\right.} \\
& \left.+\frac{\beta_{0.006-0.007}}{3.5}+\frac{\beta_{0.007-0.008}}{4.0}+\ldots\right] \frac{A}{a}
\end{aligned}
$$

where we have not taken more terms because their corresponding areas are negligible (even $A_{0.007-0.008}$ $\left.=0.07 A_{0.002-0.007}\right)$. The values of $\beta_{C_{1}-C_{2}}$ are $\beta_{0.002-0.003}$ $=0.193, \beta_{0.003-0.004}=0.090, \beta_{0.004-0.005}=0.061, \beta_{0.005-0.006}$ $=0.048, \beta_{0.006-0.007}=0.041$ and $\beta_{0.007-0.008}=0.031$. We call 
the coefficient of $A / a$ on the RHS of Eq. (86) $\kappa$. For the case of the initial LIGO, therefore, $\kappa=0.233$. Similarly, for the case of the advanced LIGO,

$$
\kappa=\frac{\beta_{0.04-0.05}}{1.25}+\frac{\beta_{0.05-0.06}}{1.5}+\frac{\beta_{0.06-0.07}}{1.75},
$$

where $\beta_{0.04-0.05}=0.183, \quad \beta_{0.05-0.06}=0.062$ and $\beta_{0.06-0.07}$ $=0.020$ which give $\kappa=0.199$. To understand what these values for $\kappa$ mean, assume that the number of templates in the remaining region of the space of interest (that is, the region with a slow variation) can be obtained by simply dividing its area by that of the unit cell. Then the total number of templates $N_{T}^{t}$ would be

$$
N_{T}^{t}=\left(1-\sum \beta_{j}\right) \frac{A}{a}+\kappa \frac{A}{a} .
$$

For the initial LIGO $\Sigma \beta_{j}=0.464$, which gives $N_{T}^{t}$ $\simeq 0.769 \mathrm{~A} / a$. Similarly for the advanced LIGO, $N_{T}^{t}$ $\simeq 0.934 \mathrm{~A} / \mathrm{a}$. This clearly shows that the variation in the area of unit cells has a small effect in the case of the advanced LIGO.

We combine the two approximations discussed above to give the following algorithm for estimating the total number of templates. Recall that in the region of large $\tau_{0}$, all the three quantities $l_{1}, l_{2}$ and $\alpha_{1}$ vary quite slowly. For the purpose of counting the number of templates, therefore, we will assume the orientation and dimensions of a unit cell to be constants in the regions (a) between vertex $A$ and the 0.002 $\mathrm{sec}^{2}$ contour for the initial LIGO and (b) between vertex $A$ and the $0.04 \mathrm{sec}^{2}$ contour for the advanced LIGO. We choose an average value of $\alpha_{1}=38^{\circ}$ for the initial LIGO and $\alpha_{1}=45^{\circ}$ for the advanced LIGO, where $\alpha_{1}$ is the angle between the semi-minor axis and the $\tau_{1.5}$ axis. The final results are quite insensitive to the choice of these angular values. In the first step of the algorithm, we count the number of templates in the region near $A$ by placing unit cells as shown schematically in Fig. 15. The unit cells are "stacked" below each other until the length of the segment along a $\tau_{0}$ $=$ const line equals $l_{2}$. Let this value of $\tau_{0}$ be $\tau_{0}^{e q}\left(l_{2}\right)$ and the number of templates thus obtained be $N_{T}^{e q}$. The area, $A_{e q}$, between the vertex $A$ and the $\tau_{0}=\tau_{0}^{e q}\left(l_{2}\right)$ line is then found. The total number of templates is then obtained as

$$
N_{T}^{t}=N_{T}^{e q}+\left[1-\frac{A_{e q}}{A}-\sum \beta_{j}\right] \frac{A}{a}+\kappa \frac{A}{a} .
$$

The output of this algorithm is shown in Fig. 16 and Fig. 17, where we have also shown the values obtained if the number of templates is estimated simply as A/a. Again, it can be seen that the effect of variation in $\mathcal{H}$ contours is small for the advanced LIGO.

Almost all the problems associated with the non-trivial shape of the boundary of the space of interest can be eliminated if instead of the segments $A B$, a rectangular corner $A D B$ were used. That is, $D$ has the abscissa of $B$ and the ordinate of $A$. However, the region between the segments $A B, A D$ and $D B$ is then mapped, in the $\left(m_{1}, m_{2}\right)$ plane, onto a negligibly small area. Thus, although the number of tem-

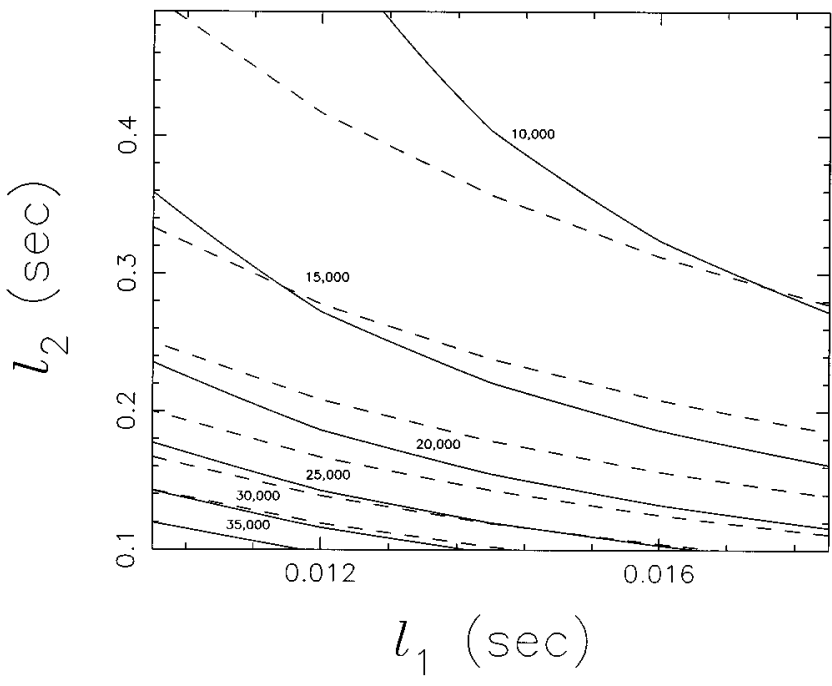

FIG. 16. The number of templates for the case of the initial LIGO as a function of the unit cell dimensions $l_{1}$ and $l_{2}$. The solid contours are obtained by using the algorithm that takes the variation of unit cell areas into account. The dashed contours are for the values obtained by simply dividing the area of the space of interest by $l_{1} \times l_{2}$.

plates will increase significantly, not much will be gained in terms of the range of detectable binary systems.

The boundary can also be made simple by going over to a different set of parameters, such as the masses $\left(m_{1}, m_{2}\right)$. But we found that in such cases the intrinsic ambiguity function shows excessive location dependence. However, there may exist a coordinate system in which both the boundary of the space of interest is simple and the intrinsic ambiguity function does not show much variation. This approach needs to be explored more thoroughly. The problems with the counting of templates, discussed above, are also present for the coordinates used in $[4,5]$.

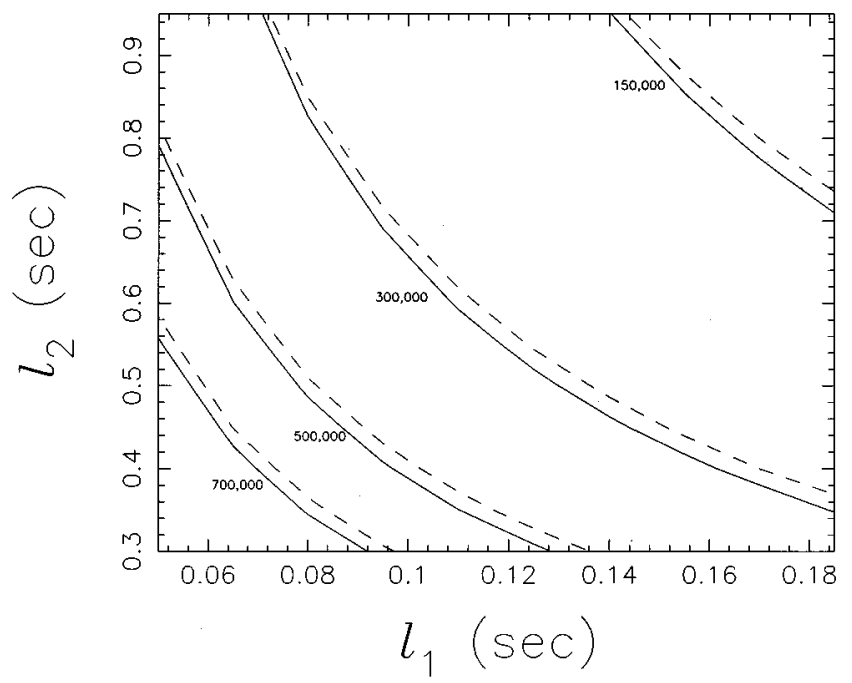

FIG. 17. The number of templates for the case of the advanced LIGO as a function of the unit cell dimensions $l_{1}$ and $l_{2}$. The solid contours are obtained by using the algorithm that takes the variation of unit cell areas into account. The dashed contours are for the values obtained by simply dividing the area of the space of interest by $l_{1} \times l_{2}$. 
To summarize, the following conclusions emerge from the discussion presented so far. (i) In the case where the intrinsic ambiguity is location independent (as happens, for instance, in the Newtonian and post ${ }^{1}$-Newtonian cases), templates should be placed on a regular grid (neglecting boundary effects). (ii) The unit cell of the grid should have the largest area [in order to satisfy (C3)] while satisfying (C1) and $(\mathrm{C} 2)$. Assuming that the unit cell is a parallelogram, we gave a practical algorithm to find the four parameters $\left(l_{1}, l_{2}, \alpha_{1}, \alpha_{2}\right)$ of this optimum unit cell. (iii) The location dependence of $\mathcal{H}$ in the post ${ }^{1.5}$-Newtonian case is quite weak over most of the space of interest (at least for the case of the advanced LIGO) as shown in Fig. 11 and Fig. 12. This implies that the placement of templates in this case should also be on an approximately regular grid. (iv) We can make a piecewise approximation to this grid where each piece, or patch, is regular (formed by translating the same unit cell). (v) If the detection probability of a signal were to be determined almost completely by the intrinsic ambiguity, then only a single unit cell for any one patch needs to be determined. We checked that this assumption is true for the post $^{1.5}$-Newtonian wave form. (vi) Since the segment $A B$ is not a strict boundary, it is acceptable if some templates from a grid near vertex $A$ breach it. We therefore put a quasiregular grid of single unit cells "stacked" vertically near $A$. In the remaining region, variation in the area of unit cells was approximately taken into account while estimating the number of unit cells. We emphasize here that these conclusions will not hold for large values of $S_{\min }$ but only for values that are sufficiently low so as to make the unit cells small.

\section{Algorithm for the determination of the optimum unit cell}

We now present the algorithm that we have used in this paper for the determination of the parameters $l_{1}$ and $l_{2}$ of the optimum one-step unit cell. Although the algorithm that was obtained earlier is practical enough, we can simplify it further as follows.

First, as has already been shown earlier (Sec. III), the threshold is very insensitive to the number of templates when the required false alarm is small. Since, as shown in Fig. 16 and Fig. 17, the relative error between the approximate count $A / a$ and the "exact" count is $\sim 30 \%$ or less, the threshold is affected negligibly if the approximate count is used in its determination.

However, it must be emphasized here that the false alarm is very sensitive to changes in the threshold and, therefore, ultimately the threshold should be fixed very accurately. For instance, let the number of templates be $3 \times 10^{5}$ and the length of a data segment be $8192 \mathrm{sec}$ with a sampling rate of $2048 \mathrm{~Hz}$ (typical values for the advanced LIGO). Then, for an average false alarm rate of 1 event/yr, the required threshold is 8.661 . If an error of, say, $-5 \%$ is made in the determination of this threshold, then the false alarm rate becomes $\sim 38$ events/yr while if the error made is $+5 \%$, then the false event rate falls to 0.02 events/yr. The latter situation is definitely more preferable, however, since the detection probability of signals will not drop too much (typically by $5 \%$ ). This in turn implies that an overestimation of the number of templates is better than an underestimation and that is precisely what happens when the approximate count of template is used (provided the effect of the boundary near vertex $A$ is negligible, which should be so for small unit cells).

Second, given a unit cell, it is sufficient to check that signals that have the least detection probability be detectable with a probability $\geqslant Q_{\mathrm{d}, \min }=0.95$. For a rectangular cell, there exist three such signals, namely, the signals at the midpoints of $l_{1}$ and $l_{2}$ and the signal at the the centroid of the rectangle. This is also borne out by Fig. 10 (lowermost) which shows that these three signals lie at the minima of the detection probability map. This is also the reason that such a set of signals was used in Figs. 13, 14.

The algorithm for one-step template placement: (i) The value of $S_{\min }$ is fixed. We make a few preliminary coarse runs to find out the range of values of $S_{\text {min }}$ for which the unit cells are sufficiently small (so that the shear is unimportant). As mentioned earlier, we keep $Q_{\mathrm{d}, \min }=0.95$ and $Q_{0, \max }$ is kept such that the average rate of false events is $1 / y r$. Let the duration of each input data segment be $T$ sec. Then,

$$
Q_{0, \max }=\left(T-\xi_{\max }\right) /(365 \times 24 \times 3600),
$$

where $\xi_{\max }$ is the duration of the longest template (see Sec. II B). Recall that we denote $T-\xi_{\max }$ by $T_{P}^{0}$.

(ii) We choose a point in the $\left(\tau_{1.5}, \tau_{0}\right)$ space such that when a unit cell is constructed around it, all the templates lie well within the boundary. For instance, in the case of the initial LIGO, we choose the point $(1.3,50.0)$. Let this point be $\theta_{a}$.

(iii) The unit eigenvectors $\hat{e}_{1 a}$ and $\hat{e}_{2 a}$ of $\mathbf{H}$ are found at $\theta_{a}$. We consider rectangular unit cells such that (a) $\theta_{a}$ is always the same vertex for all of them and (b) the sides are $l_{1} \hat{e}_{1 a}$ and $l_{2} \hat{e}_{2 a}$ with $l_{1}>0, l_{2}>0$. The values of $l_{1}$ and $l_{2}$ are chosen to lie on a regular grid in $\left(l_{1}, l_{2}\right)$ space. Typically, we keep (a) $l_{1} \in(0.01,0.05) \mathrm{sec}$ and $l_{2} \in(0.05,0.2) \mathrm{sec}$ for the initial LIGO (b) $l_{1} \in(0.04,0.1) \mathrm{sec}$ and $l_{2} \in(0.2,0.6) \mathrm{sec}$ for the advanced LIGO. The number of grid points is kept at $\sim 10 \times 10$.

(iv) Given a point $\left(l_{1}, l_{2}\right)$, the threshold $\eta$ required to get a false alarm $Q_{0, \max }$ is computed using Eq. (62). The total number of rectified output samples is $N_{r}=N_{T}^{t} \times T_{P}^{0} \times 2048$, where $T_{P}^{0}$ is the padding in the time series of the template with the longest duration (see Sec. II B) and $N_{T}^{t}$ is the total number of templates which is taken as

$$
N_{T}^{t}=\frac{\text { area of the space of interest } A}{\text { area of the unit cell }=l_{1} l_{2}} .
$$

(v) For each unit cell we consider the signal points $S_{1}$ $=\theta_{a}+\left(l_{1} / 2\right) \hat{e}_{1 a}, S_{2}=\theta_{a}+\left(l_{2} / 2\right) \hat{e}_{2 a}$ and $S_{3}=\theta_{a}+\left(l_{1} / 2\right) \hat{e}_{1 a}$ $+\left(l_{2} / 2\right) \hat{e}_{2 a}$. Their respective detection probabilities $Q_{\mathrm{d}, 1}$, $Q_{\mathrm{d}, 2}$ and $Q_{\mathrm{d}, 3}$, for the threshold $\eta$, are computed using an algorithm based on a multivariate Gaussian joint density (see Sec. III). The set $\mathcal{Z}$ is chosen from among the rectified outputs of the templates at $\theta_{a}, \theta_{a}+l_{1} \hat{e}_{1 a}, \theta_{a}+l_{2} \hat{e}_{2 a}, \theta_{a}$ $+l_{1} \hat{e}_{1 a}+l_{2} \hat{e}_{2 a}, \theta_{a}+l_{1} \hat{e}_{1 a}-l_{2} \hat{e}_{2 a}$ and $\theta_{a}-l_{1} \hat{e}_{1 a}+l_{2} \hat{e}_{2 a}$. The last two templates are included in order to take care of any contribution to the detection probability due to the shear in $\mathcal{H}$ contours. 
(vi) If the minimum among $\left\{Q_{\mathrm{d}, 1}, Q_{\mathrm{d}, 2}, Q_{\mathrm{d}, 3}\right\}$ is larger than $Q_{\mathrm{d} \text {,min }}$, then the point $\left(l_{1}, l_{2}\right)$ is recorded or else not. Let the set of unit cells which qualify thus be $L$.

(vii) The unit cell with the largest area among $L$ is chosen as the optimum unit cell.

We do not proceed further to find a larger nonrectangular unit cell because for the values of $S_{\min }$ used, there will not be much of an improvement.

\section{TWO STEP HIERARCHICAL SEARCH}

Our scheme for a two-step hierarchical search involves the use of two banks of templates, in both of which we use the same family of templates. In one of the banks, templates are spaced sparsely in (intrinsic) parameter space and this is called the first stage bank. The second stage template bank consists of templates placed more finely. The detector output is first processed through the first stage templates and the locations of those templates are noted in whose rectified outputs there was at least one crossing of a threshold $\eta_{1}$ (the first stage threshold). The value of $\eta_{1}$ is kept sufficiently low so that, even though the templates are sparsely spaced, all signals (with strength greater than some $S_{\min }$ ) can produce at least one crossing in a nearby template with a probability of $\sim 0.95$. In the next step, for each of the first stage templates that produce a crossing of $\eta_{1}$, the detector output is processed through a neighborhood of second stage templates around it. The maximum over these second stage outputs is then compared with a second stage threshold $\eta_{2}$ to check for a detection. In this way a significant saving in computation occurs since the number of templates used on the whole is much less than if the second stage bank alone were used.

The first stage templates cannot be spaced too coarsely, however, because $\eta_{1}$ has to be lowered and at some point the number of crossings because of noise alone becomes large. Since each such a false crossing would involve the use of second stage templates, the computational cost starts rising if the first stage templates are spaced too coarsely. Thus, there is a non-trivial optimization problem that needs to be solved while setting up a two-step hierarchy.

It was shown in MD96 that the correlations between templates allows a false event (crossing of $\eta_{2}$ due to noise alone) to slip through the hierarchy in spite of the presence of two thresholds. This is essentially because of the fact that the hierarchy is designed to allow the easy passage of a signal, and if a noise realization is such as to produce a crossing of $\eta_{2}$, which is quite high (typically $\sim 8.0$ ), then it would have sufficient "resemblance" to some signal (in its phase information) to allow it to pass through the hierarchy [24]. Stated in another way, this is because, for a first stage template and its neighborhood of second stage templates, the crossing of $\eta_{2}$ is not statistically independent of a crossing of $\eta_{1}$. This implies that the second stage template bank and threshold should be determined in the same way as a onestep bank for the given values of $S_{\min }, Q_{\mathrm{d}, \min }$ and $Q_{0, \max }$. The function of a two-step hierarchy is, therefore, limited to providing an estimate of the location of the global maximum. For this it utilizes the information that the occurrence of a (high) threshold crossing must generate in templates that are relatively far away from it.

We give here a brief review of the algorithm used to set up a two-step hierarchical search in the Newtonian case. Let the spacing between consecutive templates (in terms of $\tau_{0}$ ) in the one-step template bank, for given $S_{\min }, Q_{\mathrm{d} \text {, min }}$ and $Q_{0 \text {, max }}$, be $\delta_{2}$. Then the first stage spacing $\delta_{1}$ is taken to be $\delta_{1}=k \delta_{2}, k=2,3, \ldots$. For each $\delta_{1}$, the first stage threshold $\eta_{1}$ was kept such that a signal with strength $S_{\min }$, lying in the middle of two consecutive templates, has a detection probability $Q_{\mathrm{d}, \min }$. The average number of false crossings among the first stage templates can then be computed which, given the number of second stage templates to employ around each first stage crossing, in turn allows the overall average computation cost to be calculated. The minimum of this cost was then found as a function of $k$.

In the present case, a similar approach can be followed to space the first stage templates as was done for the Newtonian case. Here, there will be two spacings to fix, namely, along the minor and major axes of the one-step unit cells, and these can be chosen as integral multiples of the corresponding one step spacings $l_{1}$ and $l_{2}$. For convenience in the following, we denote a first stage unit cell at the location $\theta_{a}$ as $U_{I}\left(k_{1}, k_{2}, \theta_{a}\right)$, where the sides of the unit cells have lengths $k_{1} \times l_{1}$ and $k_{2} \times l_{2}$. There are, however, a few complications that arise in this approach. First, if a first stage unit cell $U_{I}\left(k_{1}, k_{2}, \theta_{a}\right)$ satisfies $(\mathrm{C} 1)$, it is not implied that a unit cell $U_{I}\left(k_{1}, k_{2}, \theta_{b}\right)$ at a different location will also do so. This is because the dimensions of a first stage unit cell would be quite large and Figs. 13 and 14 show that the error in detection probability rises with an increase in the dimensions. Thus the same $\left(k_{1}, k_{2}\right)$ at two different locations would lead to different values of $\eta_{1}$ since the first stage threshold is determined by the detection probability. We take this effect into account as follows. For each $\left(k_{1}, k_{2}\right)$, we take two widely separated locations $\theta_{a}$ and $\theta_{b}$ and compute the thresholds $\eta_{1 a}$ and $\eta_{1 b}$ that are required to make both $U_{I}\left(k_{1}, k_{2}, \theta_{a}\right)$ and $U_{I}\left(k_{1}, k_{2}, \theta_{b}\right)$ satisfy (C1). We then choose the minimum among these two as the first stage threshold $\eta_{1}$.

The second complication is the boundary near vertex $A$ of the space of interest which also disallows the same $\left(k_{1}, k_{2}\right)$, as in the broader parts of the space of interest, from being used in this region. Recall that the one step template grid in this region was constructed out of single unit cells "stacked" vertically (see Fig. 15). Hence, if $k_{2}>1$, extra second stage templates would be required in the region to the right of $A B$ which would increase the number of one-step templates without adding significantly to the range of binary masses being detected. However, note that if $U_{I}\left(k_{1}, k_{2}, \theta_{a}\right)$, for $k_{2}>1$, satisfies (C1) for some threshold $\eta_{1}$, then so would $U_{I}\left(k_{1}, 1, \theta_{a}\right)$, since the templates at the vertices will now be closer to all the signals in the cell's interior. Therefore, while calculating the number of first stage templates in this region, we simply divide the number of one step templates by $k_{1}$.

Finally, the number of second stage templates that would be employed per first stage crossing will now depend on whether the crossing occurs in the narrow region near vertex $A$ or in the broader part of the space of interest. Let this number be $\tilde{n}$. In the broader part of the space of interest,

$$
\tilde{n}=4\left(k_{1}-1\right)\left(k_{2}-1\right)+2\left(k_{1}-1\right)+2\left(k_{2}-1\right) .
$$


Now, the minimum of the computational cost of a two-step search occurs when the number of false crossings in the first stage becomes $\sim 1$. But most of the first stage templates will be located in the broader part of the space of interest and, hence, most of the false crossings will also occur in this region. This implies that $\tilde{n}$ will be as given in Eq. (92) for most cases. We therefore take $\tilde{n}$ to be the above for all crossings. Note that this assumption would lead to an overestimate of the computational requirements for the first stage and is, thus, "safe" in this sense.

Let the number of false crossings for a given input data segment be $n_{c}$. Then the total number of templates which will be employed for that data segment would be $n_{t}^{(1)}$ $+n_{c} \tilde{n}$, where $n_{t}^{(1)}$ is the total number of first stage templates. In the presence of a signal, there would be an extra term of $\tilde{n}$ in the above sum, but since the event rate of signals is expected to be quite low, this term can be neglected. If we assume that the first stage rectified output are all statistically independent of each other, as would be the case if they are spaced widely apart, then the average number of false crossings $n_{c}^{\text {av }}$ would be

$$
n_{c}^{\mathrm{av}}=n_{c} \times Q_{0}\left(\eta_{1}\right) .
$$

$Q_{0}\left(\eta_{1}\right)$ is the probability of at least one crossing of $\eta_{1}$ in a single rectified output [see Eq. (62)],

$$
Q_{0}(x)=1-\exp \left[-T_{P}^{0} \nu_{s} \exp \left(-x^{2} / 2\right)\right],
$$

where $\nu_{s}$ is the sampling rate and $T_{P}^{0}$ was defined in Sec. II B to be the padding in the time series of the template wave form having the longest duration. Note that since $\eta_{1}$ would be $\sim 6.0$, the effective statistical independence of rectified output samples may be less and $\epsilon$ should be less than unity. However, keeping $\epsilon=1$ leads to an overestimation of $n_{c}^{\text {av }}$ and, hence, an underestimation of the computational advantage of a two step search.

The average total computational cost for a two-step search would be

$$
n_{t}^{\mathrm{av}}=n_{t}^{(1)}+n_{c}^{\mathrm{av}} \times \tilde{n} .
$$

In order to compare the performance of a two-step search with the corresponding one-step search (that is, for the same $S_{\text {min }}, Q_{d \text {, min }}$ and $Q_{0, \text { max }}$ ), we use the computational powers required for implementing the two strategies on line (that is, the input data should be processed in the same time as required in its collection). The number of floating point operations required in a one-step search to process $T$ sec of data can be estimated as follows: (i) The number of flop involved in the discrete Fourier transform (DFT) $\tilde{x}$ of the detector output time series $\bar{x}$ would be $3 N \log _{2} N$ where $N=\nu_{s} T$. However, this transform needs to be computed only once and, thus, does not contribute significantly to the total computational cost. (ii) For each template location $\theta_{a}$, two correlations would be required, namely, with the quadrature components $\bar{q}_{0}$ and $\bar{q}_{\pi / 2}$. This involves computing the product of $\tilde{x}$ with the DFTs of $\bar{q}_{0}$ and $\bar{q}_{\pi / 2}$ followed by an inverse DFT for each of the resulting series. Hence, $2 N$ $+6 N \log _{2} N$ flop will be required here. (iii) Each of these transformed series would then have to be squared and added but only the first $T_{P}^{0} \sec$ of each series is required. This, thus, leads to $3 T_{P}^{0} \nu_{s}$ flop. Thus, the total number of operations, $N_{\text {flop }}$, involved in a one step search is

$$
N_{\text {flop }}=N_{T}^{(t)} \times\left(2 N+6 N \log _{2} N+3 T_{P}^{0} \nu_{s}\right) .
$$

For an on-line one-step search, $N_{\text {flop }}$ operations would have to be performed in $T_{P}^{0}$ sec. Thus,

$$
C_{\text {online }}^{(1)}=\frac{N_{\text {flop }}}{T_{P}^{0}} \times 10^{-9} \text { Gflops. }
$$

A similar estimate for an on-line two step search leads to an average computational requirement of

$$
C_{\mathrm{online}}^{(2)}=\frac{N_{\text {flop }}^{(2)}}{T_{P}^{0}} \times 10^{-9} \mathrm{Gflops}
$$

where

$$
N_{\text {flop }}^{(2)}=n_{t}^{\mathrm{av}} \times\left(2 N+6 N \log _{2} N+3 T_{P}^{0} \nu_{s}\right) .
$$

We call the quantity $C_{\text {online }}^{(1)} / C_{\text {online }}^{(2)}$ the computational advantage $C_{\text {gain }}$ of a two-step search. This is the factor by which a two-step hierarchical search would be faster than the corresponding one-step search in an on-line detection.

We now present our results in the form of Table I, for the initial LIGO, and Table II for the advanced LIGO. The value of $S_{\text {min }}$ for each table has been taken sufficiently low so that the resulting one-step unit cells obtained are small. It was shown in MD96 that, for a given number of templates, the one-step threshold is almost independent of $T$ for low false alarms. This implies that the unit cell dimensions will also be independent of $T$ (the variation of the threshold in the advanced LIGO case is larger but it is still negligible). Therefore, the values of $l_{1}$ and $l_{2}$, for the one-step unit cell, are given in the caption of each table. These values are for a unit cell located at $(1.3,25.0) \mathrm{sec}$ for the case of the initial LIGO and $(13.0,1000.0) \mathrm{sec}$ for the advanced LIGO. The values of the one-step threshold (which is the second stage threshold $\eta^{(2)}$ for the two-step search) and the total number of onestep templates (obtained by taking the variation of unit cell areas into account) are also given.

The first column in each table is the value of $T$. Since the sampling rate used in our calculation is $2^{11}=2048 \mathrm{~Hz}$ and a FFT is most efficient when the number of samples is a power of 2, we choose $T$ to be a power of 2 also. The second and third columns are the values of $k_{1}$ and $k_{2}$ at which the average computational cost of the two-step search is minimized. The fourth column is the corresponding first stage threshold $\eta^{(1)}$ and the fifth and sixth columns are the corresponding values of $n_{c}^{\text {av }}$ and $n_{t}^{\text {av }}$. We have kept only the integral part of $n_{c}^{\text {av }}$ and $n_{t}^{\text {av }}$ and, therefore, $n_{c}^{\text {av }}=0$ means that $n_{c}^{\text {av }} \sim 1$ or less. The seventh column is the computational power required for an on-line two-step search followed by the computational 
power required for an on-line one-step search in the eigth column. The last column lists $C_{\text {gain }}$.

Even though we have used large values of $T$, especially in Table II, such values would be difficult to use in a practical implementation because of memory restrictions. We have used these values only to show the existence of a minimum in the computational power requirement as a function of $T$ [25]. It should be noted here that for the case of the advanced LIGO, the storage of the pre-computed template wave forms is also a significant problem. For instance, even if we consider the average duration of templates in the advanced LIGO case to be $\sim 100 \mathrm{sec}$, the amount of storage required for all the $\sim 6 \times 10^{5}$ quadrature Fourier transforms would be $\sim 100 \times 2048 \times 6 \times \times 8 / 10=983$ Gbytes (assuming that each sample value requires 8 bytes of storage). This is a very low bound since the duration of a significant number of templates will be much larger.

The results obtained above can be checked approximately as follows. The one-step template placement criterion of [4] requires the templates to be placed such that, for any signal, $\mathcal{H}=0.97$ in at least one nearby template. Then the number of one-step templates $N_{T}^{t}$ would be the area $A$ of the space of interest divided by the area of the 0.97 contour. For the advanced LIGO, $N_{T}^{t}=20389.5 / 0.04 \simeq 509739$. Thus, the threshold $\eta^{2}$ required, for a false alarm rate of 1 false event/yr, would be $\eta^{(2)}=8.722$ for $T=8192.0 \mathrm{sec}$. For the detection probability formula used in MD96, it was found that the minimum observed strength required for a signal so that its detection probability be 0.95 is $S_{\mathrm{obs}} \approx \eta^{(2)}+0.67$. The actual strength should, therefore, be $S_{\text {min }}=S_{\text {obs }} / 0.97=9.682$. Roughly speaking, the decrease in $n_{t}^{\text {av }}$, with an increase in $k_{1}$ and $k_{2}$, is halted when $n_{c}^{\text {av }}$ becomes of order unity. Assuming that the number of first stage templates that is finally obtained is $\sim 10^{4}$, it would imply that, for the above value of $T$, $\eta^{(1)} \approx 7.026$. For a detection probability of 0.95 in the first stage, therefore, the value of $S_{\text {obs }}=S_{\min } \mathcal{H}^{\prime}$ should be 7.697, where $\mathcal{H}^{\prime}$ is the value of the intrinsic ambiguity in the middle of the sides of a first stage unit cell, i.e., $\mathcal{H}^{\prime}$ $=\mathcal{H}\left(\theta_{a}, \theta_{a}+k_{i} l_{i} \hat{e}_{i a} / 2\right)$. The quantity $k_{i}$ can then be calculated as the ratio of the dimension of the $\mathcal{H}^{\prime}$ contour along $\hat{e}_{i a}$ to $l_{i}\left(0.97, \theta_{a}\right)$. From the above, $\mathcal{H}^{\prime}=0.79$ which gives $k_{1}=7.67, k_{2}=4.35$ (we have allowed $k_{i}$ to be non-integral here). These values are about the same as those in Tables II. However, this approximation is crude in many ways and can only serve as an indicator for the kind of values one may get for $k_{i}$.

The savings in computational requirements achieved by a two-step search can be more than what is obtained here if the first stage template grid is rotated relative to the second stage grid. This is because of the shear of the contours. In the argument given above, the quantities $k_{1}$ and $k_{2}$ were obtained as the ratios of $l_{i}\left(0.97, \theta_{a}\right)$ and the corresponding dimension of the lower level contour $\mathcal{H}^{\prime}$. However, the direction in which the $\mathcal{H}^{\prime}$ contour is most elongated is different from that of the eigenvectors $\hat{e}_{i a}$. If the first stage grid were oriented along the direction of maximal elongation of $\mathcal{H}^{\prime}$, the first stage unit cell may turn out to be larger. However, the calculation of the number of first stage templates as well as the number, $\tilde{n}$, of second stage templates would be more involved in such a case. We postpone an investigation of this problem to a later work.

\section{CONCLUSIONS}

We have investigated the performance of a two step hierarchical search for the detection of gravitational wave signals emitted during the inspiral of a compact binary. This work extends the investigations of MD96 [6] to the more realistic case of zero spin post ${ }^{1.5}$-Newtonian template and signal wave forms.

As in MD96, we find that a two-step search brings about a significant reduction in computational requirements. For the case of (i) the initial LIGO noise PSD, a two-step search is $\sim 27.0$ times faster than the corresponding one-step search, and (ii) for the advanced LIGO noise PSD, a two-step search is $\sim 23.0$ times faster than the corresponding one-step search. The range used for the masses $m_{1}$ and $m_{2}$ is $0.5 \leqslant m_{1}$ $\leqslant 30.0 M_{\odot}, 0.5 \leqslant m_{2} \leqslant 30.0 M_{\odot}$.

In the analysis of MD96, the dominant problem was the calculation of detection probability in the presence of strong statistical correlations between rectified output samples. A solution to this problem was found in this paper in the form of a semi-analytic method that reproduces the exact Monte Carlo estimates quite well. It is also shown here that statistical correlations are unimportant for the calculation of false alarm probability when the threshold is kept sufficiently high. Therefore, the effective sampling rate used in MD96 is not required.

Though the issues of detection and false alarm probabilities have been addressed satisfactorily here, some new problems crop up in the present analysis, namely, the (i) location dependence of the intrinsic ambiguity function and (ii) the non-trivial shape of the boundary of the space of interest. Both these problems were dealt with by making some approximations. The location dependence of the intrinsic ambiguity function seems weak enough, at least in the case of the advanced LIGO, for us to assume that the grid of onestep templates will be an "adiabatically" changing regular grid. This allows us to approximately take the effect of variations in the area of the contours into account. The non-trivial boundary has a significant effect only near one of the vertices (vertex $A$ of Fig. 1). We take this effect into account by placing a single "stack" of unit cells in this region.

The results of this paper show that the use of hierarchical methods of detection can be very useful for the case of coalescing binary signals and provide a strong motivation for more detailed investigations. Such methods would be indispensable if the number of signal parameters required becomes large. For instance, if the orbital and total angular momenta of the binary are misaligned, there would be significant modulations of the phase and amplitude which can reduce the signal to noise ratio if these effects are neglected in the template family. For such signals, a template family with a larger number of parameters may be required.

Many other hierarchical strategies are also conceivable and it remains to be seen whether they can be more effective than the two-step search analyzed here. For instance, one obvious strategy is to use a lower order template family as the first stage of the search and use the true wave forms, 
having a larger number of parameters, as the second stage. It is not enough, though, to only provide estimates of their performance since at some stage such strategies need to be implemented in practice and, as seen in this paper, the details of the implementation can also be an involved issue. Also, the robustness of the placement configuration against changes in the noise power spectral density needs to be investigated. The efficacy of hierarchical methods (not necessarily a two-step search) should also be investigated for the detection of continuous wave sources where the estimated computational requirements are extremely large and far beyond presently available computing power. Further investigations in this direction are in progress.

\section{ACKNOWLEDGMENTS}

I thank Professor S. V. Dhurandhar for many helpful discussions. I acknowledge the support provided by the Council of Scientific and Industrial Research (CSIR) of India while this work was being completed. I thank Caltech and the LIGO project for hospitality.

\section{APPENDIX: THE BIVARIATE PROBABILITY DENSITY $P_{Z_{1}, Z_{2}}$ AND $\overline{Z_{1} Z_{2}}$}

Here, we outline the steps in the derivation of Eq. (64). The algebraic manipulations were performed using MATHEMATICA. First, the general expression for the joint bivariate probability density is derived without assuming the mean values of the Gaussian components to be zero. Let the bivariate cumulative distribution function of $Z_{1}=\left[X_{1}^{2}\right.$ $\left.+X_{2}^{2}\right]^{1 / 2}$ and $Z_{2}=\left[Y_{1}^{2}+Y_{2}^{2}\right]^{1 / 2}$ be $F_{Z_{1}, Z_{2}}\left(z_{1}, z_{2}\right)$, where $\left(X_{1}, X_{2}, Y_{1}, Y_{2}\right)$ is a set of jointly Gaussian random variables with a covariance matrix give in Eq. (48) and mean values $\bar{X}_{1}=\mu_{1}, \bar{X}_{2}=\mu_{2}, \bar{Y}_{1}=\nu_{1}, \bar{Y}_{2}=\nu_{2}$. Changing the variables of integration to $X_{1}=R \cos \phi, X_{2}=R \sin \phi, Y_{1}=Q \cos \psi$ and $Y_{2}=Q \sin \psi$, we get

$$
\begin{aligned}
F_{Z_{1}, Z_{2}}\left(z_{1}, z_{2}\right)= & \frac{A}{2 \pi} \int_{0}^{z_{2}} d Q \int_{0}^{2 \pi} d \psi \int_{0}^{z_{1}} d R \int_{0}^{2 \pi} d \phi R Q \exp \left[-\frac{1}{2} \frac{R^{2}+Q^{2}}{\left[1-\left(r^{2}+s^{2}\right)\right]}\right] \exp \left[\frac { Q } { 1 - ( r ^ { 2 } + s ^ { 2 } ) } \left[\left(\nu_{1}-r \mu_{1}+s \mu_{2}\right) \cos \psi\right.\right. \\
& \left.\left.+\left(\nu_{2}-s \mu_{1}-r \mu_{2}\right) \sin \psi\right]\right] \exp \left[\frac{R}{1-\left(r^{2}+s^{2}\right)}\left[\left(\mu_{1}-r \nu_{1}-s \nu_{2}\right) \cos \phi+\left(\mu_{2}+s \nu_{1}-r \nu_{2}\right) \sin \phi\right]\right] \\
& \times \exp \left[\frac{R Q}{1-\left(r^{2}+s^{2}\right)}(r \cos \phi \cos \psi+r \sin \phi \sin \psi)\right]
\end{aligned}
$$

where

$$
A=\frac{1}{2 \pi \operatorname{det}[\mathbf{C}]^{1 / 2}} \exp \left[-\frac{1}{1-\left(r^{2}+s^{2}\right)}\left(\frac{1}{2}\left(\mu_{1}^{2}+\mu_{2}^{2}+\nu_{1}^{2}+\nu_{2}^{2}\right)-r\left(\mu_{1} \nu_{1}+\mu_{2} \nu_{2}\right)-s\left(\mu_{1} \nu_{2}-\mu_{2} \nu_{1}\right)\right)\right]
$$

Equation (A1) can be rewritten as

$$
\begin{aligned}
F_{Z_{1}, Z_{2}}\left(z_{1}, z_{2}\right)= & \frac{A}{2 \pi} \int_{0}^{z_{2}} d Q \int_{0}^{2 \pi} d \psi \int_{0}^{z_{1}} d R \int_{0}^{2 \pi} d \phi R Q \exp \left[-\frac{1}{2} \frac{R^{2}+Q^{2}}{\left[1-\left(r^{2}+s^{2}\right)\right]}\right] \exp \left[\frac{Q E \cos \left(\psi+\chi_{1}\right)}{1-\left(r^{2}+s^{2}\right)}\right] \\
& \times \exp \left[\frac{R \cos \left(\phi+\chi_{3}\right)}{1-\left(r^{2}+s^{2}\right)}\left[\left(r^{2}+s^{2}\right) Q^{2}+2 D Q \sqrt{r^{2}+s^{2}} \cos \left(\psi+\chi_{2}\right)+D^{2}\right]^{1 / 2}\right],
\end{aligned}
$$

where

$$
\begin{aligned}
& E=\left[\left(\nu_{1}-r \mu_{1}+s \mu_{2}\right)^{2}+\left(\nu_{2}-r \mu_{2}-s \mu_{1}\right)^{2}\right]^{1 / 2}, \\
& D=\left[\left(\mu_{2}-r \nu_{2}+s \nu_{1}\right)^{2}+\left(\mu_{1}-r \nu_{1}-s \nu_{2}\right)^{2}\right]^{1 / 2}, \\
& \chi_{1}=\arctan \left[\frac{r \mu_{1}-s \mu_{2}-\nu_{1}}{r \mu_{2}+s \mu_{1}-\nu_{2}}\right], \\
& \chi_{2}=\arctan \left[\frac{r \mu_{1}-s \mu_{2}-\nu_{1}\left(r^{2}+s^{2}\right)}{r \mu_{2}+s \mu_{1}-\nu_{2}\left(r^{2}+s^{2}\right)}\right] .
\end{aligned}
$$

The integral over $\phi$ can be performed to yield 


$$
\begin{aligned}
F_{Z_{1}, Z_{2}}\left(z_{1}, z_{2}\right)= & A \int_{0}^{z_{2}} d Q \int_{0}^{z_{1}} d R R Q \int_{0}^{2 \pi} d \psi \exp \left[-\frac{1}{2} \frac{R^{2}+Q^{2}}{\left[1-\left(r^{2}+s^{2}\right)\right]}\right] \exp \left[\frac{Q E \cos \left(\psi+\chi_{1}\right)}{1-\left(r^{2}+s^{2}\right)}\right] \\
& \times I_{0}\left[\frac{R}{1-\left(r^{2}+s^{2}\right)}\left[\left(r^{2}+s^{2}\right) Q^{2}+2 D Q \sqrt{r^{2}+s^{2}} \cos \left(\psi+\chi_{2}\right)+D^{2}\right]^{1 / 2}\right],
\end{aligned}
$$

where $I_{0}(x)$ is the modified Bessel function of the first kind of order zero. The probability density function, $P_{Z_{1}, Z_{2}}$, can be obtained now as

$$
\begin{aligned}
P_{Z_{1}, Z_{2}}(u, v)= & \frac{\partial^{2} F_{Z_{1}, Z_{2}}(u, v)}{\partial u \partial v}=u v A \exp \left[-\frac{1}{2} \frac{u^{2}+v^{2}}{\left(1-\left(r^{2}+s^{2}\right)\right)}\right] \int_{0}^{2 \pi} d \psi \exp \left[\frac{v E \cos \left(\psi+\chi_{1}\right)}{1-\left(r^{2}+s^{2}\right)}\right] \\
& \times I_{0}\left[\frac{u}{1-\left(r^{2}+s^{2}\right)}\left[\left(r^{2}+s^{2}\right) v^{2}+2 D Q \sqrt{r^{2}+s^{2}} \cos \left(\psi+\chi_{2}\right)+D^{2}\right]^{1 / 2}\right] .
\end{aligned}
$$

In the absence of a signal, $\mu_{1}=\mu_{2}=\nu_{1}=\nu_{2}=0$ and the joint probability density reduces to

$$
P_{Z_{1}, Z_{2}}(u, v)=\frac{u v}{\sqrt{\operatorname{det} \mathbf{C}}} \exp \left[-\frac{u^{2}+v^{2}}{2\left[1-\left(r^{2}+s^{2}\right)\right]}\right] I_{0}\left[\frac{u v \sqrt{r^{2}+s^{2}}}{1-\left(r^{2}+s^{2}\right)}\right] .
$$

Thus, the correlation $\overline{u v}$ can be obtained as

$$
\overline{u v}=\int_{0}^{\infty} \int_{0}^{\infty} d u d v \frac{u^{2} v^{2}}{1-\left(r^{2}+s^{2}\right)} \exp \left[-\frac{u^{2}+v^{2}}{2\left[1-\left(r^{2}+s^{2}\right)\right]}\right] I_{0}\left[\frac{u v \sqrt{r^{2}+s^{2}}}{1-\left(r^{2}+s^{2}\right)}\right] .
$$

The above double integral is solved in [26] from which we get

$$
\overline{u v}=2 \mathbf{E}\left[\sqrt{r^{2}+s^{2}}\right]-\left[1-\left(r^{2}+s^{2}\right)\right] \mathbf{K}\left[\sqrt{r^{2}+s^{2}}\right],
$$

where $\mathbf{E}$ is a complete elliptic integral of the second kind and $\mathbf{K}$ is a complete elliptic integral of the first kind.

[1] A. Abramovici, W. E. Althouse, R. W. P. Drever, Y. Gursel, S. Kawamura, F. J. Raab, D. Shoemaker, L. Sievers, R. E. Spero, K. S. Thorne, R. E. Vogt, R. Weiss, S. E. Whitcomb, and M. E. Zucker, Science 256, 325 (1992).

[2] C. Bradaschia, et al. Nucl. Instrum. Methods Phys. Res. A 289, 518 (1990).

[3] K. S. Thorne, in 300 Years of Gravitation, edited by S. W. Hawking and W. Israel (Cambridge University Press, Cambridge, England, 1987).

[4] B. Owen, Phys. Rev. D 53, 6749 (1996).

[5] T. A. Apostolatos, Phys. Rev. D 54, 2421 (1996).

[6] S. D. Mohanty and S. V. Dhurandhar, Phys. Rev. D 54, 7108 (1996).

[7] T. A. Apostolatos, Phys. Rev. D 52, 605 (1995).

[8] L. S. Finn and D. F. Chernoff, Phys. Rev. D 47, 2198 (1993).

[9] C. Cutler and E. E. Flanagan, Phys. Rev. D 49, 2658 (1994).

[10] C. W. Helstrom, Statistical Theory of Signal Detection, 2nd ed. (Pergamon, London, 1968).

[11] A. Krolak, J. A. Lobo, and B. J. Meers, Phys. Rev. D 43, 2470 (1991).

[12] S. V. Dhurandhar and B. S. Sathyaprakash, Phys. Rev. D 49, 1707 (1994).

[13] W. H. Press, B. P. Flannery, S. A. Teukolsky, and W. T. Vetterling, Numerical Recipes (Cambridge University Press, Cambridge, England, 1986).
[14] L. Milano, F. Barone, and M. Milano, Phys. Rev. D 55, 4537 (1997).

[15] R. N. Bracewell, Fourier Transform and Its Applications, 2nd ed. (McGraw-Hill, New York, 1986).

[16] O. E. Brigham, Fast Fourier Transform and Its Applications (Prentice-Hall, Englewood Cliffs, NJ, 1988).

[17] A. Stuart and J. K. Ord, Kendall's Advanced Theory of Statistics, 5th ed. (Oxford University Press, New York, 1987), Chap. 15.

[18] R. Y. Rubinstein, Simulation and the Monte Carlo Method (Wiley, New York, 1981).

[19] Let the number of samples in each time series be be $N$ and the number of templates be $M$. We use the following formula for the number of flop for each realization:

$$
\left[2 N+2\left(3 N \log _{2} N\right)+3 N\right] \times M,
$$

where the first term from the left is for the computation of the products of Fourier transforms (the factor 2 is for the two quadrature filters), the second is for their inverse transforms and the third for squaring and adding the outputs.

[20] D. Knuth, The Art of Computer Programming (AddisonWesley, Reading, MA, 1981), Vol. 2.

[21] S. O. Rice, Bell Syst. Tech. J. 23, 282 (1944); 24, 46 (1945); reprinted in Selected Papers on Noise and Stochastic Processes, edited by Nelson Wax (Dover, New York, 1954). A 
brief derivation of the relevant expressions is also given in [10], Chap. 9, Sec. 2.

[22] J. W. Goodman, Statistical Optics (Wiley, New York, 1985).

[23] The contours in Figs. 5,6 and Figs. 7,8 were obtained by mapping the corresponding contours obtained on a regular grid in $\left(m_{1}, m_{2}\right)$ space. It is not possible to use a uniform grid of points in $\left(\tau_{1.5}, \tau_{0}\right)$ space because of the nontrivial boundary. To obtain $\delta_{i}$, we interpolate on the regular grid in $\left(m_{1}, m_{2}\right)$ and take the cross section of this interpolating function along the desired curve (say, a constant $\tau_{0}$ curve).

[24] B. F. Schutz (private communication).

[25] B. F. Schutz, in The Detection of Gravitational Radiation, edited by D. Blair (Cambridge University Press, Cambridge, England, 1989), pp. 406-427.

[26] A. P. Prudnikov et al., Integrals and Series, 3rd ed. (Gordon and Breach, New York, 1992), Vol. 2, Chap. 3. 